\title{
Well-Posedness and Long-Time Dynamics of Geophysical Fluid Flows
}

\author{
Maleafisha Joseph Pekwa Stephen Tladi \\ rica
}

${ }^{1}$ Department of Mathematics and Applied Mathematics University of Limpopo Private Bag 1106 Polokwane, 0727 South Af-

\begin{abstract}
The author elucidates in a concrete way dynamical challenges concerning approximate inertial manifolds (AIMS), i.e., globally invariant, exponentially attracting, finite-dimensional smooth manifolds, for nonlinear dynamical systems on Hilbert spaces. The goal of this theory is to prove the basic theorem of approximation dynamics, wherein it is shown that there is a fundamental connection between the order of the approximating manifold and the well-posedness and long-time dynamics of the rotating Boussinesq and quasigeostrophic equations.
\end{abstract}

\section{Keywords}

Rotating Boussinesq and Quasigeostrophic Equations, Geophysical Fluid Flows, Well-Posedness and Long-Time Dynamics, Existence and Uniqueness, Stability and Attraction, Approximate Inertial Manifolds. 


\section{Contents}

Chapter 1. Models For The Rotating Boussinesq Equations With Reynolds Stress . . . . . . . . . . 293

1.1 Description for the rotating Boussinesq equations with Reynolds stress . . . . . . . . . . . . . 293

1.2 The initial-boundary value problems for the rotating Boussinesq equations with Reynolds

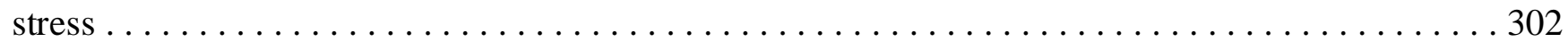

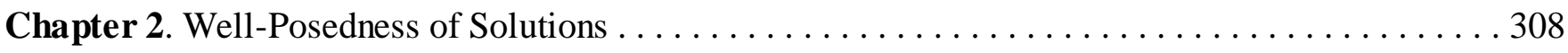

2.1 Existence and uniqueness of solutions to the rotating Boussinesq equations with Reynolds

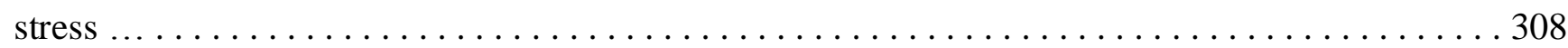

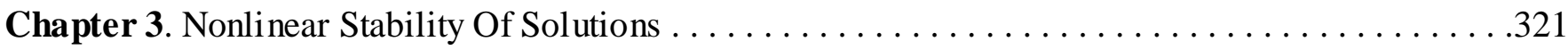

3.1 Aspects of stability and attraction for the rotating Boussinesq equations with Reynolds stress ... 321 


\section{Chapter 1. Models for the Rotating Boussinesq Equations with Reynolds Stress}

\subsection{Description for the rotating Boussinesq equations with Reynolds stress}

The objective of this investigation is to develop prototype geophysical fluid dynamics models as the first effort towards understanding the impact of rotating Boussinesq equations in the ocean. First, we review the fundamental assumptions and techniques involved in the derivation of a system of partial differential equations governing geophysical fluid flows $[6,10,39]$. The evolution equations for the quantities of interest, which may be scalar or vector or tensor valued are derived with the assistance of Reynolds' transport theorem, conservation laws and constitutive assumptions [4, 6, 22]. The basic fields in the description of the motion and states of geophysical phenomena are the velocity, the pressure, the density, the temperature and the salinity. The equations governing these fields consist of the mass balance or continuity equation, the momentum equation, the energy equation and the equation for salt budget.

Consider a geophysical fluid occupying a region $B \in \mathbb{R}^{3}$ for the benchmark Lagrangian coherent structures utilizing the DsTool package program of Worfolk, Guckenheimer et al; [66] which greatly helped codify the discipline.

For clarity, conventional vector, tensor and indicial notation are utilized. Also, for convenience in all that follows, we will adopt a Cartesian coordinate system on a spherical rotating earth. The x-axis is directed westward, the $y$-axis is southward and the z-axis is oriented upward.

Heuristically, Reynolds' transport theorem asserts that the rate at which the integral of density for a geophysical fluid parcel over $V(t)$ is changing is equal to the sum of the rate performed with $V(t)$ fixed in its current position and the rate at which density is transported out of the fluid parcel $V(t)$ across its boundary. Consequently, the use of the divergence theorem to transform the surface integral into a volume integral gives the equation

$$
\frac{\partial \rho}{\partial t}+\underline{u} \cdot \nabla \rho+\rho \nabla \cdot \underline{u}=0,
$$

for mass balance where $\underline{u}=(u, v, w)$ is Eulerian velocity of the fluid, $\rho$ is density, $\mathrm{t}$ is time, and $\underline{x}=(x, y, z)$ is the coordinate system. Equation 1:1 is the continuity equation for compressible fluids. Similarly, in order to obtain the equation of motion we utilize Reynolds' transport theorem, and the conservation hypothesis that the rate of change of momentum transported in a geophysical fluid parcel of volume $\mathrm{V}(\mathrm{t})$ is balanced by the resultant of both body and surface forces. Concerning the contact or surface forces, as in the analysis of the relative motion of a fluid near a point and Cauchy's constitutive hypothesis, we represent surfaces forces by the stress tensor $\sigma$. Further, it is instructive to decompose the velocity gradient $5 \mathrm{u}$ into the superposition of symmetric rate-of-deformation tensor $\mathrm{D}$ and the skew-symmetric vorticity tensor $\Omega$ defined by 


$$
\begin{aligned}
D & =\frac{1}{2}\left(\nabla \underline{u}+\nabla^{T} \underline{u}\right) \\
\Omega & =\frac{1}{2}\left(\nabla \underline{u}-\nabla^{T} \underline{u}\right) .
\end{aligned}
$$

Next, we consider the Cauchy's stress tensor $\sigma$ using the constitutive assumption

$$
\sigma=-p+2 \mu\left(D-\frac{1}{3} \nabla \cdot \underline{u}\right)
$$

for a Newtonian viscous compressible fluid. Here $\mu$ is dynamic viscosity and $\mathrm{p}$ is pressure. In addition, consideration of the important ambient rotation of geophysical fluids and application of the simplifying effect of the continuity equation yield the following equation for the conservation of linear momentum customarily called the equation of motion

$$
\rho\left(\frac{\partial \underline{u}}{\partial t}+\underline{u} \cdot \nabla \underline{u}+\underline{f} \times \underline{u}+g \underline{k}\right)=-\nabla p+\mu \triangle \underline{u}+\frac{\mu}{3}(\nabla \cdot \underline{u}),
$$

where $\mathrm{g}$ is gravitation, $f=\left(0, f, f_{*}\right)$ is the earth's rotation, $f$ is Coriolis parameter and $f_{*}$ is reciprocal Coriolis parameter. Scale analysis ensures consideration of the meriodinal change in the Coriolis parameter and the reciprocal Coriolis parameter. According to the investigations [10,39], nonlinear Rossby waves and vortex coherent structures such as alternating cyclones and anticyclones of the Gulf stream and Agulhas current span numerous degrees of latitude; and for them, it is imperative to consider the meridional change in the Coriolis parameter $f$ and the reciprocal Coriolis parameter $f_{*}$. The $\beta$-plane approximation to the Coriolis $f$ and the reciprocal Coriolis $f_{*}$ is obtained by considering $\frac{\mathrm{y}}{\mathrm{r}_{0}}$ as sufficiently small and invoking the 2-term Taylor series

$$
\begin{gathered}
f=f_{0}+\beta_{0} y, \\
f_{*}=r_{0} \beta_{0}-\frac{f_{0}}{r_{0}} y,
\end{gathered}
$$

where $f_{0}=2 \varpi \sin \varphi_{0}$ is the Coriolis parameter at reference altitude $\varphi_{0} ; y=\left(\varphi-\varphi_{0}\right) r_{0}$ is the coordinate oriented southward, $\varpi$ is the angular rate for rotating framework of reference, $r_{0}$ is the earth's radius and $\beta_{0}=2 \varpi r_{0}^{-1} \cos \varphi_{0}$ is the beta parameter. We note that the $\beta$-plane approximation is valid whenever the term $\beta_{0} y$ is small compared to the leading term $f_{0}$. The Cartesian coordinate system where the beta term $\beta_{0} y$ is not retained is called the f-plane approximation, that is,

$$
\begin{gathered}
f=2 \varpi \sin \varphi_{0}, \\
f_{*}=2 \varpi \cos \varphi_{0} .
\end{gathered}
$$

The inclusion of the distinguishing geophysical rotary term in the balance of momentum asserts that the inertial acceleration of geophysical fluids is the decomposition of the relative acceleration and the Coriolis acceleration due to the rotating framework of reference. The significance of the Coriolis rotary term is in the generation of planetary or Rossby 
waves that support geophysical jets and mesoscale vortices. And the validity of the approximation (1.5) is a consequence of restriction to geophysical phenomena with length scales substantially smaller than the radius of the earth [6, 10,39]. Consequently, no appeal will be made in this investigation to the spherical geometry of the earth which in curvilinear coordinate system contains challenging extraneous curvature terms. Formal passage from the $\beta$-plane approximation to retain the extraneous curvature terms corresponding to the full geometry of the spherical rotating earth is treated in [6, 39]. The mathematical analysis of the resulting geophysical fluid equations with extraneous curvature terms is examined in Lions et al $[62,61]$.

Equations (1.1) - (1.4) are supplemented by equation of state, energy and salt budgets. In order to derive these results, utility of the first law of thermodynamics and Reynolds's transport theorem assert that the rate of change of the internal energy supplied to a geophysical fluid parcel is balanced by the heat out of the fluid parcel and the power or work done by the system against external forces. Thus, by consideration of the power produced by the surfaces forces and Fourier's consitutive hypothesis for the rate of heat and subsequent application of the continuity equation give the following temperature form of the energy equation

$$
\rho C_{v}\left(\frac{\partial T}{\partial t}+\underline{u} \cdot \nabla T\right)+p \nabla \cdot \underline{u}=\kappa_{T} \Delta T+\mu \nabla \cdot(\underline{u} \cdot \nabla \underline{u})-\frac{2}{3} \mu(\nabla \cdot \underline{u})^{2}
$$

An alternative derivation of the energy equation may be achieved by exploiting the enthalpy relation [4].

The equations must be completed with the addition of the state equation

$$
\rho=\rho_{0}\left[1-\alpha_{T}\left(T-T_{0}\right)+\alpha_{S}\left(S-S_{0}\right)\right]
$$

and the following salt budget

$$
\frac{\partial S}{\partial t}+\underline{u} \cdot \nabla S=\kappa_{S} \Delta S
$$

which asserts that ocean water conserve their salt content except in the presence of diffusion. In the evolution equations (1.7) - (1.9), $\mathrm{T}$ is absolute temperature, $C v$ is heat capacity, $K \mathrm{~T}$ is thermal conductivity, $\mathrm{S}$ is salinity, $T_{0}, S_{0}, \rho_{0}$ are reference values of temperature, salinity and density, $\alpha T$ is coefficient of thermal expansion, $\alpha \mathrm{S}$ is coefficient of saline contraction and $K S$ is coefficient of saline diffusion. The coupled equations (1.1) - (1.9) yield the following system of partial differential equations governing general heterogenous compressible geophysical fluid flows: 


$$
\begin{aligned}
\frac{\partial \rho}{\partial t}+\underline{u} \cdot \nabla \rho+\rho \nabla \cdot \underline{u} & =0, \\
\rho\left(\frac{\partial \underline{u}}{\partial t}+\underline{u} \cdot \nabla \underline{u}+\underline{f} \times \underline{u}+g \underline{k}\right) & =-\nabla p+\mu \Delta \underline{u}+\frac{\mu}{3}(\nabla \cdot \underline{u}), \\
\rho C_{v}\left(\frac{\partial T}{\partial t}+\underline{u} \cdot \nabla T\right)+p \nabla \cdot \underline{u} & =\kappa_{T} \Delta T+\mu \nabla \cdot(\underline{u} \cdot \nabla \underline{u})-\frac{2}{3} \mu(\nabla \cdot \underline{u})^{2}, \\
\rho & =\rho_{0}\left[1-\alpha_{T}\left(T-T_{0}\right)+\alpha_{S}\left(S-S_{0}\right)\right], \\
\frac{\partial S}{\partial t}+\underline{u} \cdot \nabla S & =\kappa_{S} \Delta S .
\end{aligned}
$$

Next, the equations for the conservation of mass, momentum, energy and salt budget are simplified by the Boussinesq assumption which states that the effect of compressiblity is negligible in the balance equation except in the buoyancy term and the equation of state.

Employing the additive decomposition

$$
\rho=\rho_{0}+\dot{\rho}(x, y, z, t), \quad \dot{\rho} \ll \rho_{0}
$$

in (1.1) and retaining terms multiplied by $\rho_{0}$, we obtain

$$
\nabla \cdot \underline{u}=0
$$

which is the continuity equation for an incompressible fluid. Physically, this statement means that conservation of mass has become conservation of volume. Another implication is the elimination of sound waves. Substitution in (1:10) of the additive decompositions (1:11) and

$$
p=p_{0}(z)+p ́(x, y, z, t)
$$

with $p_{0}(z)=P 0-\rho_{0} g z$ being the hydrostatic pressure, taking into account the continuity equation (1.12) and retaining dominating terms, we obtain the set of equations for a general heterogeneous incompressible geophysical fluid flows:

$$
\begin{aligned}
\nabla \cdot \underline{u} & =0, \\
\rho_{0}\left(\frac{\partial \underline{u}}{\partial t}+\underline{u} \cdot \nabla \underline{u}+\underline{f} \times \underline{u}\right)+g \rho \underline{k} & =-\nabla p+\mu \Delta \underline{u}, \\
\rho_{0} C_{v}\left(\frac{\partial T}{\partial t}+\underline{u} \cdot \nabla T\right) & =\kappa_{T} \Delta T, \\
\rho & =\rho_{0}\left[1-\alpha_{T}\left(T-T_{0}\right)+\alpha_{S}\left(S-S_{0}\right)\right], \\
\frac{\partial S}{\partial t}+\underline{u} . \nabla S & =\kappa_{S} \Delta S .
\end{aligned}
$$


We set $\kappa_{T}=\frac{\kappa_{T}}{\rho_{0} C_{v}}$; and $\nu=\frac{\mu}{\rho_{0}}$ is the kinematic viscosity.

In order to circumvent the challenges of directly accounting for molecular diffusion and oceanic salt finger pattern formations that result from the competitive effects of the diffusivities of heat and salt budgets, we examine the case where the salt and heat diffusivities are assumed to be equal to the eddy diffusivity, that is, $\kappa=\kappa_{T}=\kappa_{S}$. The choice of the eddy diffusivity $[10,39,37,38,1,5,16,17,56,58,55,57,28,27,64,63,45,46,47,21,7,8,9,13,33,34,35,65$, $59,31]$, which forms the basis of the model analyzed here, incorporates the ubiquitous geophysical phenomena that temperature, density and salinity structures of the ocean are influenced primarily by chaotic transport and mixing within jet streams and geophysical eddying currents on the scale of the Rossby deformation radius. The Rossby deformation radius is defined as the distance covered by a wave travelling at a given speed during one inertial period. The existence and persistence of jet streams and other geophysical coherent structures in the ocean make plausible that molecular diffusion is weak to be directly significant in the evolution equations governing the observed organized motions of a viscous incompressible stratified fluid with the Coriolis force.

Application of (1.11) into the equation of state (1.8) and applying the operators $\Delta$ and $\frac{\partial}{\partial t}+\underline{u} \cdot \nabla$ and taking (1.13) in consideration yield

$$
\frac{\partial \dot{\rho}}{\partial t}+\underline{u} \cdot \nabla \dot{\rho}=\kappa \Delta \dot{\rho} .
$$

Thus, dropping the primes from $\rho$ and $p$, the above Boussinesq approximation gives the following system of primitive partial differential equations

$$
\begin{aligned}
\rho_{0}\left(\frac{\partial \underline{u}}{\partial t}+\underline{u} \cdot \nabla \underline{u}+\underline{f} \times \underline{u}\right)+g \rho \underline{k} & =-\nabla p+\mu \triangle \underline{u}, \\
\nabla \cdot \underline{u} & =0, \\
\frac{\partial \rho}{\partial t}+\underline{u} \cdot \nabla \rho & =\kappa \Delta \rho .
\end{aligned}
$$

These equations govern the flow of a viscous incompressible stratified fluid with the Coriolis force. Some preliminary remarks are offered on other geophysical fluid dynamics models that are derived from the equations (1.15) where use is made of a perturbation analysis of the flow fields at the Rossby number on the order of unity or less. In particular, at the zero-order we obtain the geostrophic equations and in this limit the rotating Boussinesq equations reduces to a balance between the Coriolis force and the pressure gradient. Subsequently, first-order terms in the Rossby number yield the quasigeostrophic equations that govern the evolution of geostrophic pressure.

Next, concerning the the boundary conditions we assume the fields to be periodic along the horizontal coordinates $x$ and $y$ with periods $L 1$ and $L 2$, respectively. The flow domain is assigned on an open rectangular periodic region $B=\left\{(x, y, z) \in\left(0, L_{1}\right) \times\left(0, L_{1}\right) \times(0, h)\right\}$ with boundary $\Gamma=\{z=0, h\}$. The domain B represents the flow region and the surfaces $z=0$ and $z=h$ represent the lower and upper boundaries of the ocean, respectively. To system (1.15) we 
prescribe boundary conditions

$$
\begin{gathered}
\frac{\partial u}{\partial z}=\frac{\partial v}{\partial z}=w=0 \text { at } \Gamma, \\
\rho(z=0)=\rho_{l} \\
\rho(z=h)=\rho_{u}
\end{gathered}
$$

where $\rho_{l}$ and $\rho_{u}$ are constants. In order to eliminate rigid motions we consider the case

$$
\int_{B} u d x=\int_{B} v d x=0
$$

We introduce the following conducting solutions

$$
\begin{array}{r}
\rho=\bar{\rho}-\rho_{l}+\frac{z}{2}\left(\rho_{u}-\rho_{l}\right) \\
p=\bar{p}+z \rho_{l} g-\frac{g}{4} z^{2}\left(\rho_{u}-\rho_{l}\right)
\end{array}
$$

for density and pressure, respectively. Substitution in (1.15) lead to the system of partial differential equations

$$
\begin{aligned}
\frac{\partial \underline{u}}{\partial t}+\underline{u} \cdot \nabla \underline{u}+\underline{f} \times \underline{u}+\frac{g}{\rho_{0}} \rho \underline{k} & =-\frac{1}{\rho_{0}} \nabla p+\nu \Delta \underline{u}, \\
\nabla \cdot \underline{u} & =0, \\
\frac{\partial \rho}{\partial t}+\underline{u} \cdot \nabla \rho+\left(\rho_{u}-\rho_{l}\right) \underline{u} \cdot \underline{k} & =\kappa \Delta \rho .
\end{aligned}
$$

We embark on the following additive decomposition of flow fields for the rotating Boussinesq equations (1.15):

$$
\begin{aligned}
& \underline{u}=\langle\underline{u}>+\underline{u}, \\
& p=\langle p>+\dot{p}, \\
& \rho=\langle\rho\rangle+\dot{\rho} .
\end{aligned}
$$

Here the time-averaging operator $<$. $>$ is defined by

$$
<\underline{u}>=\frac{1}{\tau} \int_{\tau} \underline{u}(\underline{x}) \chi(\underline{x}) d \underline{x}
$$

with $T$ being a characteristic evolution time-scale and $\chi(\underline{x})$ a probability density function. The terms in the splitting (1.18) represent coherent and incoherent flow fields, respectively. 
Furthermore, we assume that the incoherent velocity field satisfies

$$
<\underline{u}>=0
$$

The formal additive decomposition of the flow fields of the rotating Boussinesq equations into coherent and incoherent terms is justified partly by the realization that solutions to the primitive equations of geophysical fluid dynamics contain both fast and time scales with frequencies proportional to the Rossby number.

Employing the decomposition (1.18) in the rotating Boussinesq equations (1.15) and invoking the time- averaging operator, the system

$$
\begin{aligned}
\rho_{0}\left(\frac{\partial \underline{u}}{\partial t}+\underline{u} \cdot \nabla \underline{u}+\underline{f} \times \underline{u}\right)+g \rho \underline{k} & =-\nabla p+\mu \Delta \underline{u}+\nabla \cdot A \\
\nabla \cdot \underline{u} & =0 \\
\frac{\partial \rho}{\partial t}+\underline{u} \cdot \nabla \rho & =\kappa \Delta \rho+\nabla \cdot W .
\end{aligned}
$$

for coherent flow fields in which $A$ and $W$ are Reynolds stress [39] fields. The presence of the effective dissipative terms $\nabla . A$ and $\nabla . W$ implies that although the incoherent flow fields have zero average, the momentum and density fluxes of the fluctions, which are quadratic in the incoherent fluctions, need not vanish when the time-averaging operator is employed. As far as the contributions of the incoherent flow fields are concerned, we observe that a prototypical fluid parcel with fluctuation velocity $u^{\prime}$ in the x-direction will transport z-momentum $\rho_{0} \dot{w}^{\prime}$ with a nonzero flux across the surface given by the quadratic relation $-\rho_{0}<\dot{u} w==A_{x z}$. It is similarly convenient to introduce the following quadratic protocols for other Reynolds stress fields 


$$
\begin{aligned}
& A_{x x}=-\rho_{0}\langle\dot{u} u ́\rangle \text {, } \\
& A_{y y}=-\rho_{0}\langle\dot{v} v \dot{ }, \\
& A_{z z}=-\rho_{0}\langle\dot{w} w \dot{w}\rangle \text {, }
\end{aligned}
$$

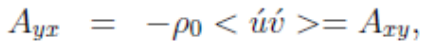

$$
\begin{aligned}
& A_{z x}=-\rho_{0}<\dot{u} w \dot{w}>=A_{x z}, \\
& A_{z y}=-\rho_{0}<\dot{v} w==A_{y z}, \\
& \left.W_{x}=-\rho_{0}<\dot{u} \rho \dot{ }\right\rangle, \\
& \left.W_{y}=-\rho_{0}<\hat{v} \dot{\rho}\right\rangle, \\
& W_{z}=-\rho_{0}\langle\dot{w} \rho \dot{\rho}\rangle .
\end{aligned}
$$

It is useful from the oceanographic and meteorological perspectives to idealize the Reynolds stress fields as a representation of wind stress quantities. To be more specific, we consider the wind stress comprising primarily the trades that is communicated to ocean water through absorption of a shear stress and is responsible for driving oceanic circulations in the form of jets and eddying currents that impact the earth's weather [10, 39]. It is important to realize that the additive decomposition of the ageostrophic flow quantities into coherent and incoherent terms and consideration of the averaging operator to obtain the Reynolds stress fields or wind stress fields have resulted in a set of evolution equations that are not closed. We adopt the following Pedlosky closure protocols [39] for the Reynolds stress fields (1.20):

$$
\begin{aligned}
& A_{x x}=-2 \varepsilon \rho_{0} \triangle^{5} \frac{\partial u}{\partial x}, \\
& A_{y y}=-2 \varepsilon \rho_{0} \triangle^{5} \frac{\partial v}{\partial y}, \\
& A_{z z}=-2 \varepsilon \rho_{0} \triangle^{5} \frac{\partial w}{\partial z}, \\
& A_{y x}=-\varepsilon \rho_{0} \triangle^{5}\left(\frac{\partial u}{\partial x}+\frac{\partial v}{\partial y}\right)=A_{x y}, \\
& A_{z x}=-\varepsilon \rho_{0} \triangle^{5}\left(\frac{\partial w}{\partial z}+\frac{\partial u}{\partial x}\right)=A_{x z}, \\
& A_{z y}=-\varepsilon \rho_{0} \triangle^{5}\left(\frac{\partial w}{\partial z}+\frac{\partial v}{\partial y}\right)=A_{y z},
\end{aligned}
$$

And 


$$
\begin{aligned}
& W_{x}=-\delta\left(\frac{\partial^{2} u}{\partial x^{2}}+\triangle^{5} \frac{\partial \rho}{\partial x}\right), \\
& W_{y}=-\delta\left(\frac{\partial^{2} v}{\partial y^{2}}+\triangle^{5} \frac{\partial \rho}{\partial y}\right), \\
& W_{z}=-\delta\left(\frac{\partial^{2} w}{\partial z^{2}}+\triangle^{5} \frac{\partial \rho}{\partial z}\right),
\end{aligned}
$$

where $\varepsilon$ and $\delta$ are parameters. The geophysical motivation of the choice of the closure protocols $(1.21-1.22)$ will become clear in the sequel when we give a mathematical treatment of the resulting evolution equations. As a validation from a dynamical systems approach, the closure protocols lead to well- posedness in the sense of Hadamard and the existence of attractors for the solution of the system of partial differential equations. The closure protocols (1.21 - 1.22) are the simplest which allows existence of stability and attraction. Another validation desirable from the craft [25, 21] of stability and attraction follows from the fact that a significant quantity in analyzing whether the solution of an evolution equation is bounded in some suitable norm is that of Lyapunov functional of the system with interpretations such as fictitious energy, enstrophy and entropy production which are decreasing along solutions. The equations governing the flow of ageostrophic equations with Reynolds stress (1.21 - 1.22) induce damping mechanisms in the nature of viscosity, diffusion, stratification and rotation effects that manifest themselves in the evolution equation with the existence of Lyapunov functionals which are equivalent to some norm induced by the inner product of solution.

We now proceed with the derivation of our geophysical fluid dynamics model. Employing the Pedlosky closure hypothesis into the equations (1.19) for coherent flow fields gives the following primitive partial differential equations governing rotating Boussinesq equations with Reynolds stress:

$$
\begin{aligned}
\rho_{0}\left(\frac{\partial \underline{u}}{\partial t}+\underline{u} \cdot \nabla \underline{u}+\underline{f} \times \underline{u}+\varepsilon \Delta^{6} \underline{u}\right)+g \rho \underline{k} & =-\nabla p+\mu \Delta \underline{u}, \\
\nabla \cdot \underline{u} & =0, \\
\frac{\partial \rho}{\partial t}+\underline{u} \cdot \nabla \rho+\delta \Delta^{6} \rho & =\kappa \Delta \rho .
\end{aligned}
$$

The presence of effective dissipative terms $\Delta^{6} \underline{\underline{u}}$ and $\Delta^{6} \rho$ is equivalent to stress due to the impact of the incoherent terms on the coherent flow. Alternatively, the dissipative terms account for the wind stress along the ocean surface. Next, concerning the boundary conditions, we assume the fields to be periodic along the coordinates $x, y$ and $z$ with periods $L 1$, $L 2$ and $L 2$, respectively. The flow domain is assigned on an open rectangular periodic region

$$
B=\left\{(x, y, z) \in\left(0, L_{1}\right) \times\left(0, L_{2}\right) \times\left(0, L_{3}\right)\right\} .
$$

To system (1.23) we prescribe the following space-periodic boundary conditions for the flow fields 


$$
\begin{aligned}
\underline{u}\left(\underline{x}+L e_{i}, t\right) & =\underline{u}(\underline{x}, t) \\
\rho\left(\underline{x}+L e_{i}, t\right) & =\rho(\underline{x}, t) \\
p\left(\underline{x}+L e_{i}, t\right) & =p(\underline{x}, t)
\end{aligned}
$$

where $\left\{e_{1}, e_{2}, e_{3}\right\}$ is the standard basis of $\mathbb{R}^{3}$ and with the simplifying assumption that the period $L=L 1=L 2=L 3$. We further assume the derivatives of $\underline{u}, \rho$ and $\mathrm{p}$ are also space-periodic. In order to eliminate rigid motions we consider the case where the average velocity and pressure vanish

$$
\begin{aligned}
& \int_{B} \underline{u}(\underline{x}, t) d x=0, \\
& \int_{B} p(\underline{x}, t) d x=0 .
\end{aligned}
$$

Substitution in (1.23) reduce to the following:

$$
\begin{aligned}
\frac{\partial \underline{u}}{\partial t}+\underline{u} \cdot \nabla \underline{u}+\underline{f} \times \underline{u}+\frac{g}{\rho_{0}} \rho \underline{k}+\varepsilon \triangle^{6} \underline{u} & =-\frac{1}{\rho_{0}} \nabla p+\nu \triangle \underline{u}, \\
\nabla \cdot \underline{u} & =0, \\
\frac{\partial \rho}{\partial t}+\underline{u} \cdot \nabla \rho+\left(\rho_{u}-\rho_{l}\right) \underline{u} \cdot \underline{k}+\delta \triangle^{6} \rho & =\kappa \Delta \rho .
\end{aligned}
$$

\subsection{The initial-boundary value problems for the rotating Boussinesq equations with Reynolds stress}

In this section we introduce the nondimensional form of the equations governing the flow of a viscous incompressible stratified fluid under the Coriolis force without Reynolds stress. In order to achieve this objective, the system of partial differential equations and boundary conditions are made nondimensional with the following length and velocity scales of motions in which rotation and stratification effects are essential: 


$$
\begin{gathered}
x=L \bar{x}, y=L \bar{y}, z=H \bar{z}, t=\frac{L}{U} \bar{t} \\
u=U \bar{u}, v=U \bar{v}, w=\frac{U H}{L} \bar{w}, L_{1}=L \bar{L}_{1} \\
L_{2}=L \bar{L}_{2}, \rho=\frac{1}{g H} f_{0} \rho_{0} U L \bar{\rho}, p=f_{0} \rho_{0} U L \bar{p} \\
\rho_{u}-\rho_{l}=\frac{1}{g} N^{2} \rho_{0}, \bar{\beta}=\frac{L \cos \varphi_{0}}{r_{0} R o \sin \varphi_{0}}
\end{gathered}
$$

where $\mathrm{T}=\frac{\mathrm{L}}{\mathrm{U}}$ represent the time scale, $U$, represent the horizontal velocity scale, $W$ represent the vertical velocity scale, $L$ represent the length scale, $H$ represent the vertical length scale, etc. Distinguishing attributes of geophysical fluid dynamics are due to the effects of rotation, density heterogeneity or stratification and scales of motion. To ensure the measure of the effect of variations of density defined by stratification frequency

$$
N^{2}=-\frac{g}{\rho_{0}} \frac{d \rho}{d z}
$$

is real(which implies static stability of the stratified fluid), we assume $\frac{d \rho}{d z}<0$ for $0 \leq \mathrm{z} \leq \mathrm{h}$. It is known that unstable density stratification leads to rapid convective motions. Employing the beta-plane approximation and substituting the above scaling into the system (1.17) and omitting bars from nondimensional quantities, we obtain the following nondimensional form of the equations governing the flow of a viscous incompressible stratified fluid with the Coriolis force:

$$
\begin{aligned}
R o\left(\frac{\partial \underline{u}}{\partial t}+\underline{u} \cdot \nabla \underline{u}\right)-\left(1+y R o \beta_{0}\right) \underline{u} \times \underline{k}+\rho \underline{k} & =-\nabla p+E k \Delta \underline{u}, \\
\nabla \cdot \underline{u} & =0, \\
\frac{F r^{2}}{R o}\left(\frac{\partial \rho}{\partial t}+\underline{u} \cdot \nabla \rho\right)+\underline{u} \cdot \underline{k} & =\frac{F r^{2}}{R o(E d)} \Delta \rho,
\end{aligned}
$$

where we set the aspect ratio $\frac{\mathrm{H}}{\mathrm{L}}$, to unity. The evolution equations have been scaled so that the relative order of each term is measured by the dimensionless parameter multiplying it. Here the parameter $R_{0}=\frac{U}{L_{0}}$

is the Rossby number which compares the inertial term to the Coriolis force; $F_{r}=\frac{U}{N H}$ is the Froude number which 
measures the importance of stratification; $\mathrm{Ek}=\frac{\mathrm{V}}{\mathrm{H}^{2} \mathrm{f}_{0}}$ measures the relative importance of frictional dissipation; $\mathrm{Ed}=\frac{\mathrm{V}}{\mathrm{f}_{0} \mathrm{~N}^{2}}$ is the nondimensional eddy diffusion coefficient. The ratio $\frac{\mathrm{Fr}^{2}}{\mathrm{R}_{0}}$ measures the significant influence of both rotation and stratification to the dynamics of the flow. The corresponding initial-boundary conditions are specified as follows: We make the vertical density boundary conditions homogeneous using

$$
\begin{array}{r}
\rho=\bar{\rho}+\rho_{l}+\frac{1}{g} z \rho_{0} N^{2} \\
p=\bar{p}-z \rho_{l}-\frac{1}{2 g} z^{2} \rho_{0} N^{2}
\end{array}
$$

Omitting bars the conditions (1.16) become

$$
\begin{gathered}
\frac{\partial u}{\partial z}=\frac{\partial v}{\partial z}=w=\rho=0 \text { at } \Gamma, \\
\int_{B} u d x=\int_{B} v d x=0 .
\end{gathered}
$$

And the flow domain reduces to $B=\{(x, y, z) \in(0, L) \times(0, L) \times(0,1)\}$ with boundary $\Gamma=\{z=0,1\}$. Furthermore, velocity and density are given at initial time $\mathrm{t}=0$

$$
\begin{aligned}
& \underline{u}(\underline{x}, 0)=u_{0}(\underline{x}) \\
& \rho(\underline{x}, 0)=\rho_{0}(\underline{x}) .
\end{aligned}
$$

We conclude this section with recapitulation of the set of equations that will be analyzed in this investigation. The evolutionary equation for the $\beta$-plane rotating Boussinesq equations governing geophysical fluid flows is the following initial-boundary value problem:

$$
\begin{gathered}
R o\left(\frac{\partial \underline{u}}{\partial t}+\underline{u} \cdot \nabla \underline{u}\right)-\left(1+y R o \beta_{0}\right) \underline{u} \times \underline{k}+\rho \underline{k}=-\nabla p+E k \Delta \underline{u}, \\
\nabla \cdot \underline{u}=0, \\
\frac{F r^{2}}{R o}\left(\frac{\partial \rho}{\partial t}+\underline{u} \cdot \nabla \rho\right)+\underline{u} \cdot \underline{k} \quad=\frac{F r^{2}}{R o(E d)} \Delta \rho, \\
\frac{\partial u}{\partial z}=\frac{\partial v}{\partial z}=w=\rho=0 \text { at } \quad \Gamma, \\
\int_{B} u d x=\int_{B} v d x=0 . \\
\underline{u}(\underline{x}, 0)=u_{0}(\underline{x}) \\
\rho(\underline{x}, 0)=\rho_{0}(\underline{x}) .
\end{gathered}
$$


In this section we introduce the nondimensional form of the equations governing the flow of a viscous incompressible stratified fluid under the Coriolis force with Reynolds stress. In order to achieve this objective, the system of partial differential equations and boundary conditions are made nondimensional with the following length and velocity scales of motions in which rotation and stratification effects are essential:

$$
\begin{gathered}
x=L \bar{x}, y=L \bar{y}, z=H \bar{z}, t=\frac{L}{U} \bar{t} \\
u=U \bar{u}, v=U \bar{v}, w=\frac{U H}{L} \bar{w}, L_{1}=L \bar{L}_{1} \\
L_{2}=L \bar{L}_{2}, \rho=\frac{1}{g H} f_{0} \rho_{0} U L \bar{\rho}, p=f_{0} \rho_{0} U L \bar{p} \\
\rho_{u}-\rho_{l}=\frac{1}{g} N^{2} \rho_{0}, \bar{\beta}=\frac{L \cos \varphi_{0}}{r_{0} \operatorname{Ros} \sin \varphi_{0}}
\end{gathered}
$$

where $\mathrm{T}=\frac{\mathrm{L}}{\mathrm{U}}$ represent the time scale, $\mathrm{U}$, represent the horizontal velocity scale, $\mathrm{W}$ represent the vertical velocity scale, $\mathrm{L}$ represent the length scale, $\mathrm{H}$ represent the vertical length scale, etc. Distinguishing attributes of geophysical fluid dynamics are due to the effects of rotation, density heterogeneity or stratification and scales of motion. To ensure the measure of the effect of variations of density defined by stratification frequency

$$
N^{2}=-\frac{g}{\rho_{0}} \frac{d \rho}{d z}
$$

is real(which implies static stability of the stratified fluid), we assume $\frac{d \rho}{d z}<0$. It is known that an unstable density stratification leads to rapid convective motions. Employing the beta-plane approximation and substituting the above scaling into the system (1.17) and omitting bars from nondimensional quantities, we obtain the following nondimensional form of the equations governing the flow of a viscous incompressible stratified fluid under the Coriolis force with Reynolds stress:

$$
\begin{aligned}
R o\left(\frac{\partial \underline{u}}{\partial t}+\underline{u} \cdot \nabla \underline{u}\right)-\left(1+y R o \beta_{0}\right) \underline{u} \times \underline{k}+\rho \underline{k}+E k \Delta^{6} \underline{u} & =-\nabla p+E k \Delta \underline{u}, \\
\nabla \cdot \underline{u} & =0, \\
\frac{F r^{2}}{R o}\left(\frac{\partial \rho}{\partial t}+\underline{u} \cdot \nabla \rho\right)+\underline{u} \cdot \underline{k}+\frac{F r^{2}}{E d(R o)} \Delta^{6} \rho & =\frac{F r^{2}}{\operatorname{Ro}(E d)} \Delta \rho,
\end{aligned}
$$

where we set the aspect ratio $\frac{\mathrm{H}}{\mathrm{L}}$ to unity. The evolution equations have been scaled so that the relative order of each term 
is measured by the dimensionless parameter multiplying it. Here the parameter $R_{0}=\frac{U}{L_{0}}$

is the Rossby number which compares the inertial term to the Coriolis force; $F_{r}=\frac{U}{N H}$ is the Froude number which measures the importance of stratification; $\mathrm{Ek}=\frac{\mathrm{V}}{\mathrm{H}^{2} \mathrm{f}_{0}}$ measures the relative importance of frictional dissipation; $\mathrm{Ed}=\frac{\mathrm{V}}{\mathrm{f}_{0} \mathrm{~N}^{2}}$ is the nondimensional eddy diffusion coefficient. The ratio $\frac{\mathrm{Fr}^{2}}{\mathrm{R}_{0}}$ measures the significant influence of both rotation and stratification to the dynamics of the flow. The corresponding initial-boundary conditions are specified as follows: The space-periodicity of the fields and the vanishing of the average velocity and pressure are represented by

$$
\begin{gathered}
\underline{u}\left(\underline{x}+L e_{i}, t\right)=\underline{u}(\underline{x}, t), \\
\rho\left(\underline{x}+L e_{i}, t\right)=\rho(\underline{x}, t), \\
p\left(\underline{x}+L e_{i}, t\right)=p(\underline{x}, t), \\
\int_{B} \underline{u}(\underline{x}, t) d x=0, \\
\int_{B} p(\underline{x}, t) d x=0,
\end{gathered}
$$

where the flow domain $B=\{(x, y, z) \in(0, L) \times(0, L) \times(0, L)\}$ is the periodic region.

Furthermore, velocity and density are given at initial time $\mathrm{t}=0$

$$
\begin{aligned}
& \underline{u}(\underline{x}, 0)=u_{0}(\underline{x}) \\
& \rho(\underline{x}, 0)=\rho_{0}(\underline{x}) .
\end{aligned}
$$

We conclude this chapter with recapitulation of the set of equations that will be analyzed in this investigation. The evolutionary equation for the $\beta$-plane rotating Boussinesq equations with Reynolds stress is the following initial-boundary value problem:

$$
\begin{aligned}
R o\left(\frac{\partial \underline{u}}{\partial t}+\underline{u} \cdot \nabla \underline{u}\right)-\left(1+y R o \beta_{0}\right) \underline{u} \times \underline{k}+\rho \underline{k}+E k \Delta^{6} \underline{u} & =-\nabla p+E k \Delta \underline{u}, \\
\nabla \cdot \underline{u} & =0, \\
\frac{F r^{2}}{R o}\left(\frac{\partial \rho}{\partial t}+\underline{u} \cdot \nabla \rho\right)+\underline{u} \cdot \underline{k}+\frac{F r^{2}}{E d(R o)} \Delta^{6} \rho & =\frac{F r^{2}}{R o(E d)} \Delta \rho,
\end{aligned}
$$




$$
\begin{aligned}
& \underline{u}\left(\underline{x}+L e_{i}, t\right)=\underline{u}(\underline{x}, t), \\
& \rho\left(\underline{x}+L e_{i}, t\right)=\rho(\underline{x}, t), \\
& p\left(\underline{x}+L e_{i}, t\right)=p(\underline{x}, t),
\end{aligned}
$$

$$
\int_{B} \underline{u}(\underline{x}, t) d x=0,
$$

$$
\begin{aligned}
& \int_{B} p(\underline{x}, t) d x=0, \\
& \underline{u}(\underline{x}, 0)=u_{0}(\underline{x}) \\
& \rho(\underline{x}, 0)=\rho_{0}(\underline{x}) .
\end{aligned}
$$




\section{Chapter 2. Well-Posedness of Solutions}

\subsection{Existence and uniqueness of solutions to the rotating Boussinesq equations with Reynolds stress}

In the functional formulation of the initial-boundary value problems, the following function spaces of divergence-free or solenoidal vector functions in the sense of the theory of distributions will be utilized:

$$
\begin{aligned}
& Q=\left\{\underline{u} \in L_{2}(B): \nabla \cdot \underline{u}=0, \underline{u} \text { satisfies Space-periodicity }\right\}, \\
& X=\left\{\mid \tau: \tau_{i j} \in L_{2}(B), \tau_{i j}=\tau_{j i}, \tau_{i j}=0 \text {, a.e. } \in B\right\} \\
& H= \begin{cases}H_{1} \times \text { Closure of } Q \text { in } L_{2}(B) & \text { Space-periodicity } \\
H_{1} \times H_{0} & \text { Condition (1.27) }\end{cases} \\
& V= \begin{cases}V_{1} \times \text { Closure of } Q \text { in } H^{1}(B) & \text { Space-periodicity } \\
V_{1} \times V_{0} & \text { Condition (1.27) }\end{cases}
\end{aligned}
$$

which are Hilbert spaces equipped, respectively, with norms $\|\cdot\|_{H} \equiv\|\cdot\|_{L^{2}},\|\cdot\|_{V} \equiv\|\cdot\|_{1}$ and $\|\cdot\|_{X} \equiv\|\cdot\|_{L^{2}}$ corresponding to the product Hilbert structure. The rigorous construction and useful features of Hilbert spaces of divergence-free or solenoidal vector functions can be found in the noteworthy and accesible work of Ladyzhenskaya [36]. Here H1 = L2(B) and V1 is the space of functions in $\mathrm{H}^{1}$ (B) satisfying condition (1.27) which are Hilbert spaces endowed, respectively, with norms $\|\cdot\|_{H_{1}} \equiv\|\cdot\|_{L^{2}}$ and $\|\cdot\|_{V_{1}} \equiv\|\cdot\|_{1}$ And the function spaces H0 and V0 are given by

$$
H_{0}=\left\{\underline{u} \in L_{2}(B): \nabla \cdot \underline{u}=0 \text { in } B, \underline{u} \cdot \mathbf{n}=0 \text { at } \Gamma\right\},
$$

with $\mathrm{n}$ the unit outward normal on the boundary $\Gamma$ and

$$
V_{0}=\left\{\underline{u} \in V_{1}: \nabla \cdot \underline{u}=0\right\}
$$

which are Hilbert spaces with norm denoted by $\|\cdot\|_{1}$. Additionally, we need the following function space of tesor-valued functions

$$
\mathbf{H}^{k}=H^{k}(B) \cap X
$$

Let $\alpha=\frac{E k}{R o}$ or $\frac{1}{E k}$. It is useful to set

$$
A_{s} \underline{u}=-\alpha \Pi \triangle \underline{u},
$$


where $\underline{u} \in D\left(A_{s}\right)$, the domain of the Stokes operator As, and $\Pi$ is the orthogonal projection of $\mathrm{L}^{2}(\mathrm{~B})$ onto H0. Due to the presence of the boundary condition (1:27) and the flow region $B=\{(x, y, z) \in(0, L) \times(0, L) \times(0,1)\}_{\text {With }}$ boundary $\Gamma=\{z=0,1\}$, we have

$$
\Pi \Delta \underline{u}=\Delta \underline{u}-\nabla \Phi
$$

with $\Phi$ satisfying the Neumann problem corresponding to the Laplace operator:

$$
\begin{gathered}
\triangle \Phi=0 \text { in } B, \\
\frac{\partial \Phi}{\partial z}=\triangle w \text { at } \Gamma .
\end{gathered}
$$

The domain of the Stokes operator As is defined by

$$
D\left(A_{s}\right)=V_{0} \cap H^{2}(B),
$$

and is endowed with the norm $\|\underline{u}\|_{D\left(A_{s}\right)}=\left\|A_{s} \underline{u}\right\|_{L^{2}}$ which is equivalent to the natural norm $\|\cdot\|_{2}$ of the Sobolev space $H^{2}(B)$. Moreover, the Laplace's operator defined by

$$
A_{l} \rho=-\alpha \triangle \rho
$$

has domain given by

$$
D\left(A_{l}\right)=V_{1} \cap H^{2}(B)
$$

In order to ensure the solution of an evolution equation is bounded in some suitable norm, we need to consider the following Hilbert space

$$
D(L)=\left\{\begin{array}{l}
\left\{U \in \Upsilon: \int_{B}\left(|\triangle \underline{u}|^{2}+|\triangle \rho|^{2}\right) d x<\infty, \text {, } \underline{u} \text { satisfy }(1.27)\right\} \\
\left\{U \in \Upsilon: \int_{B}\left(\left|\triangle^{6} \underline{u}\right|^{2}+\left|\triangle^{6} \rho\right|^{2}\right) d x<\infty, \underline{u} \text { satisfy }(1.30)\right\} .
\end{array}\right.
$$

We recall that the Stokes operator As and the Laplace's operator Al may be regarded as ubounded self-adjoint positive linear operators, respectively, from $D(A s)$ into $H O$ and from $D(A l)$ into $H l$ defined by

$$
\begin{aligned}
& \left(A_{s} \underline{u}, \underline{v}\right)=((\underline{u}, \underline{v}))=((\underline{u}, \underline{v}))_{1}, \\
& \left(A_{l} \underline{u}, \underline{v}\right)=((\underline{u}, \underline{v}))=((\underline{u}, \underline{u}))_{1}
\end{aligned}
$$

for all $\underline{u}, \underline{v} \in D\left(A_{l}\right)$ and $D\left(A_{s}\right)$. Furthermore, $A_{l}^{-1}$ and $A_{s}^{-1}$ are compact self-adjoint linear operators in $H 1$ and $H 0$. The basic function space $D\left(L^{n}\right)$ is Hilbert space with norm $\|U\|_{D\left(L^{n}\right)}=\left\|L^{n} U\right\|$. Further, the dual of $D\left(L^{\frac{1}{2}}\right)$ is denoted by $D\left(L^{\frac{-1}{2}}\right)$. Under appropriate assumptions $[50,49,48,14]$, it is possible to establish existence of the follow- 
ing injections

$$
D\left(L^{\frac{1}{2}}\right)=V \subset D(L) \subset \Upsilon=H \subset D\left(L^{\frac{-1}{2}}\right) .
$$

The density and compactness of the injections will find applications in the sequel when we prove well-posedness of solutions to the initial-boundary value problems $(1.29$ - 1.31) and (1.26 - 1.28). In addition to the Stokes operator and Laplace operator, with the assistance of the orthogonal projection $\Pi$ we will need the bilinear mapping b(., .) defined by

$$
\mathbf{b}(\underline{u}, \underline{v})= \begin{cases}\Pi(\underline{u} \cdot \nabla) \underline{v}=\mathbf{b}_{s}(\underline{u}, \underline{v}) & \text { Condition }(1.27) \\ (\underline{u} \cdot \nabla) \underline{v}=\mathbf{b}_{l}(\underline{u}, \underline{v}) & \text { Space-periodicity condition }\end{cases}
$$

where $\underline{u}, \underline{v} \in D\left(A_{s}\right)$ for the bilinear operator $\mathbf{b}_{s}(\underline{u}, \underline{v})$ and $\underline{u} \in D\left(A_{s}\right), \underline{v} \in D\left(A_{l}\right)$ for the bilinear operator $\mathbf{b}_{l}(\underline{u}, \underline{v})$.

The goal of this section is to establish results of existence, uniqueness and differentiability of the solutions for the initial-boundary value problem of $\beta$-plane rotating Boussinesq equations with Reynolds stress $(1.29-1.31)$. We prove the significant aspects of wellposedness of solution utilizing the machinery developed above and additional accessible techniques constructed in this section. The functional formulation for the evolution equation of $\beta$-plane rotating Boussinesq equations with Reynolds stress (1.29-1.31) takes the following form of an initial value problem in the Hilbert space $\Upsilon$ :

$$
\begin{aligned}
\frac{d U}{d t}+L U+N(U) & =0 \\
U(0) & =U_{0}
\end{aligned}
$$

where $U=(u, v, w, \rho)$. The boundary conditions have been taken to be space-periodic in order to simplify the analysis. Concerning the linear operator $L U$ and the nonlinear operator $N(U)$ we have 


$$
\begin{aligned}
& N(U)=B(U, U)+C U-F U \\
& F U=-\left(0,0, \frac{\rho}{R o}, \frac{R o}{F r^{2}} w\right) \\
& C U=-\left(\begin{array}{l}
\frac{E k}{R o} \triangle \underline{u} \\
\frac{1}{E d} \triangle \rho
\end{array}\right)=\left(\begin{array}{l}
A_{l} \underline{u} \\
A_{l} \rho
\end{array}\right) \\
& B(U, U)=B(U)=\left(\begin{array}{l}
\underline{u} \cdot \nabla \underline{u} \\
\underline{u} \cdot \nabla \rho
\end{array}\right)=\left(\begin{array}{l}
\mathbf{b}_{l}(\underline{u}, \underline{u}) \\
\mathbf{b}_{l}(\underline{u}, \rho)
\end{array}\right) \\
& L U=T U+S U \\
& T U=-\left(\begin{array}{l}
\frac{E k}{R o} \triangle^{6} \underline{u} \\
\frac{1}{E d} \triangle^{6} \rho
\end{array}\right)=\left(\begin{array}{l}
A_{l}^{6} \underline{u} \\
A_{l}^{6} \rho
\end{array}\right) \\
& S=-\left(\frac{1+y \beta R o}{R o}\right)\left(\begin{array}{cccc}
0 & -1 & 0 & 0 \\
1 & 0 & 0 & 0 \\
0 & 0 & 0 & 0 \\
0 & 0 & 0 & 0
\end{array}\right) \forall U \in D(L)=D(T) \text {. }
\end{aligned}
$$

In order to develop basic results concerning whether there exists a unique solution in the large for the general three-dimensional space variables for initial-value problem (2.2), we derive a priori energy type estimates useful in proving that (2.2) generate a dynamical system, denoted, $U(t)=S(t) U 0$, where $U(t)$ is the unique solution uniformly bounded in time and $S(t)$ is a group of continuous nonlinear solution operators. The principal result concerning the existence of such a unique solution will be proven by the utility of the Faedo-Galerkin technique, a priori energy type estimates and Leray-Schauder principle. The linear operator LU of the initial-value problem (2.2) satisfies

$$
(L U, U)=(T U, U) \equiv a(U, U)
$$

where $\mathrm{a}(.$, . .) is symmetric bilinear form. It follows from $[50,19,36,32,2,30,29]$ that this implies $\mathrm{L}$ is self-adjoint. Additionally,

$$
(L U, U)=(T U, U) \geq \lambda\|U\|^{2}>0
$$

which implies the bilinear form induced by $L$ is coercive and $L^{-1}$ is compact and self-adjoint. By the courtesy of an excellent result from $[50,19,36,32], \lambda$ is the smallest eigenvalue of Laplace's operator and Stokes operator. Also, the symmetry of the bilinear form a $(U, W)$, together with the coerciveness of a $(U, U)$ imply the existence of a sequence of positive eigenvalues and a corresponding sequence of eigensolutions which yield an orthonormal basis of $\Upsilon$ such that 


$$
\begin{gathered}
L W_{i}=\lambda_{i} W_{i} \\
0<\lambda_{1} \leq \lambda_{2} \leq \ldots \leq \lambda_{i} \leq \ldots,
\end{gathered}
$$

and $\lambda_{i} \rightarrow \infty$ as $i \rightarrow \infty$. Moreover,

$$
\begin{gathered}
\left\|L^{\frac{1}{2}} U\right\| \geq \lambda_{1}^{\frac{1}{2}}\|U\| \\
\|L U\| \geq \lambda_{1}^{\frac{1}{2}}\left\|L^{\frac{1}{2}} U\right\|
\end{gathered}
$$

The following useful inequalities are utilized in the derivation of a priori energy type estimates:

$$
\begin{gathered}
(B(U), U)=\int_{\Omega}[(\underline{u} \cdot \nabla \underline{u} \cdot \underline{u})+(\underline{u} \cdot \nabla \rho \rho] d x=0 \\
\|C U\|^{2} \leq \lambda_{1}\left\|L^{\frac{1}{2}} U\right\|^{2} \leq \lambda_{1}^{\frac{1}{2}}\|L U\|\left\|L^{\frac{1}{2}} U\right\|
\end{gathered}
$$

Since $L C=C L$, the inequality

$$
\left\|L^{\frac{1}{2}} C U\right\|^{2} \leq \lambda_{1}\|L U\|^{2}
$$

holds. By the courtesy of the Sobolev inequality and continuity properties of the nonlinearity $B(U)$ the following bounds are satisfied

$$
\begin{gathered}
\|B(U)\|^{2} \leq c_{0}^{2}(C U, U)\left[\|C U\|^{2}+\|U\|^{2}\right] \\
\leq c_{0}^{2}\left(\lambda_{1}+1 / \lambda_{1}\right)\|U\|\left\|L^{\frac{1}{2}} U\right\|^{2}\|L U\|
\end{gathered}
$$

By the virtue of Cauchy-Schwarz inequality, Young's inequality and (2.10) the following is satisfied

$$
|(B(U)+C U, L U)| \leq 54\left(c_{1}^{4}\|U\|^{2}\left\|L^{\frac{1}{2}} U\right\|^{2}+\lambda_{1}\right)\left\|L^{\frac{1}{2}} U\right\|^{2}+\frac{1}{4}\|L U\|^{2}
$$

Where $c_{1}=c_{0}^{2}\left(\lambda_{1}+1 / \lambda_{1}\right)$. Also, from [14] the following a priori estimates is valid

$$
\left\|L^{\frac{1}{2}} B(U)\right\|^{2} \leq c\|L U\|^{2} .
$$

Concerning the solution to the steady linear case we have

$$
\begin{gathered}
(L U+C U, U)=(T U, U)+(C U, U) \\
\geq\left(\lambda+\lambda_{1}^{\frac{1}{6}}\right)\|U\|^{2}>0,
\end{gathered}
$$

which implies $L+C$ is positive and hence the bilinear form it induces is coercive. This establishes existence and uniqueness of solution to the steady linear case by the Riesz-Frechet representation theorem or the Lax-Milgram lemma. The following mini-max principle from $[50,15,41,42,43,44,18,23,24,25]$ will be utilized:

$$
\lim _{t \rightarrow \infty} \sup \|F\|^{2} \leq\left(\frac{R o}{F r^{2}}+\frac{1}{R o}\right)^{2}\|\Omega\|^{2}
$$


Where $\|\Omega\|$ is the measure of $\Omega$. Taking the inner product of (2.2) with $U$, and invoking the inequalites (2.13), (2.6) and Young's inequality yield the following differential inequality:

$$
\begin{aligned}
& \frac{d}{d t}\|U\|^{2}+\alpha \lambda_{1}\|U\|^{2} \\
\leq & \frac{d}{d t}\|U\|^{2}+\alpha\left\|L^{\frac{1}{2}} U\right\|^{2} \\
\leq & \frac{1}{\alpha}\left(\frac{R o}{F r^{2}}+\frac{1}{R o}\right)^{2}\|\Omega\|^{2}
\end{aligned}
$$

Where $\alpha=\lambda+\lambda_{1}^{\frac{1}{6}}$. The consideration of Gronwall's inequality in (2.15) provides a priori estimate

$$
\|U(t)\|^{2} \leq\left\|U_{0}\right\|^{2} \exp (-\alpha t)+\frac{1}{\alpha^{2}}\left(\frac{R o}{F r^{2}}+\frac{1}{R o}\right)^{2}\|\Omega\|^{2}(1-\exp (-\alpha t))
$$

which is asymptotic stability with ow energy and entropy production provided by

$$
E(t)=\frac{1}{2}\|U(t)\|^{2}=\frac{1}{2} \int_{\Omega}\left(\underline{u}^{2}+\rho^{2}\right) d x
$$

and exponential dissipation rate $\alpha=\lambda+\lambda_{1}^{\frac{1}{6}}$. Thus, we are able to assert that the following asymptotic estimate is valid

$$
\lim _{t \rightarrow \infty} \sup \|U(t)\|^{2} \leq \frac{1}{\alpha^{2}}\left(\frac{R o}{F r^{2}}+\frac{1}{R o}\right)^{2}\|\Omega\|^{2}
$$

which shows $U(t)$ is uniformly bounded in time in $\Upsilon$. Taking the inner product of (2.2) with $L U$ and using inequalities (2.6) and (2.11), we find that

$$
\begin{gathered}
\frac{d}{d t}\left\|L^{\frac{1}{2}} U\right\|^{2}+\lambda_{1}\left\|L^{\frac{1}{2}} U\right\|^{2} \\
\leq \frac{d}{d t}\left\|L^{\frac{1}{2}} U\right\|^{2}+\|L U\|^{2} \\
\leq 2\left(\frac{R o}{F r^{2}}+\frac{1}{R o}\right)^{2}\|\Omega\|^{2}+108\left(c_{1}^{4}\|U\|^{2}\left\|L^{\frac{1}{2}} U\right\|^{4}+\lambda_{1}\left\|L^{\frac{1}{2}} U\right\|^{2}\right)
\end{gathered}
$$

We estimates (2.15) using the inequality

$$
\lim _{t \rightarrow \infty} \sup \int_{t}^{t+1}\left\|L^{\frac{1}{2}} U(s)\right\|^{2} d s \leq\left(1 / \alpha^{2}+1 / \alpha^{3}\right)\left(\frac{R o}{F r^{2}}+\frac{1}{R o}\right)^{2}\|\Omega\|^{2}
$$

and by the virtue of Gronwall's inequality in (2.18) we obtain a priori estimate

$$
\left\|L^{\frac{1}{2}} U(t)\right\|^{2} \leq \rho_{1}^{2}
$$

where $\rho_{1}^{2}=\nu_{1} \nu_{2}$, and the parameters $\nu_{1}$ and $\nu_{2}$ are given by the following involved and tedious expressions, respectively,

$$
\nu_{1}=\left(2+107 \lambda_{1}\left(1 / \alpha^{2}+1 / \alpha^{3}\right)+1 / \alpha^{2}+1 / \alpha^{3}\right)\left(\frac{R o}{F r^{2}}+\frac{1}{R o}\right)^{2}\|\Omega\|^{2}
$$


And

$$
\nu_{2}=\exp \left(108 c_{0}^{2}\left(1 / \alpha^{2}+1 / \alpha^{3}\right)\left(\frac{R o}{F r^{2}}+\frac{1}{R o}\right)^{2}\|\Omega\|^{2}\right) .
$$

Consequently from the last inequality the following is valid

$$
\lim _{t \rightarrow \infty} \sup \left\|L^{\frac{1}{2}} U(t)\right\|^{2} \leq \rho_{1}^{2}
$$

which shows $U(t)$ is uniformly bounded in time in $D\left(L^{\frac{1}{2}}\right)$. Similarly, taking the inner product of (2.2) with $L^{2} U$ and making use of the inequality (2.6) and (2.12) yield

$$
\begin{gathered}
\frac{d}{d t}\|L U\|^{2}+\lambda_{1}\|L U\|^{2} \\
\leq \frac{d}{d t}\|L U\|^{2}+\left\|L^{\frac{3}{2}} U\right\|^{2} \\
\leq 3 c^{2}\|L U\|^{4}+3 \lambda_{1}\|L U\|^{2}+3\left(\frac{1}{R o^{2}}+\frac{R o^{2}}{F r^{4}}\right) \rho_{1}^{2}
\end{gathered}
$$

Using the differential inequality (2.18) and invoking Holder inequality we obtain a priori energy type estimate

$$
\int_{t}^{t+1}\|L U(s)\|^{2} d s \leq \nu_{3}^{2}
$$

Where $\nu_{3}^{2}=a_{1}+a_{2}$ denotes the sum of the involved expressions

$$
a_{1}=\lambda_{1}\left(1 / \alpha^{2}+1 / \alpha^{3}\right)\left(\frac{R o}{F r^{2}}+\frac{1}{R o}\right)^{2}\|\Omega\|^{2}+\rho_{1}^{2}
$$

And

$$
a_{2}=108 c_{0}^{2}\left(1 / \alpha^{2}+1 / \alpha^{3}\right)^{2}\left(\frac{R o}{F r^{2}}+\frac{1}{R o}\right)^{4}\|\Omega\|^{4} .
$$

Substitution of this a priori energy type estimate into (2.21) yields

$$
\|L U(t)\|^{2} \leq \rho_{2}^{2}
$$

with $\rho_{2}^{2}$ denoting

$$
\rho_{2}^{2}=3 c^{2} \nu_{3}^{4}+2 \lambda_{1} \nu_{3}^{2}+3\left(\frac{1}{R o^{2}}+\frac{R o^{2}}{F r^{4}}\right) \rho_{1}^{2} .
$$

Consequently, putting the estimates together we obtain

$$
\lim _{t \rightarrow \infty} \sup \|L U(t)\|^{2} \leq \rho_{2}^{2}
$$

which shows $U(t)$ is uniformly bounded in time in $D(L)$. We now state existence, uniqueness, continuity and differentiability with respect to initial conditions of solution to the initial-value problem (2.2) and invoke the above a priori estimates to prove the result. 
Proposition 2.1.1 Under the above hypothesis for $F \in D\left(L^{\frac{1}{2}}\right)$ and $U_{0} \in \Upsilon$ given, (2.2) generate a dynamical system $U(t)=S(t) U O$ satisfying

$$
U \in C([0, T] ; \Upsilon) \cap L^{2}\left(0, T ; D\left(L^{\frac{1}{2}}\right)\right)
$$

for all $T>0$. Moreover, if $U_{0} \in D\left(L^{\frac{1}{2}}\right)$ then

$$
U \in C\left([0, T] ; D\left(L^{\frac{1}{2}}\right)\right) \cap L^{2}(0, T ; D(L))
$$

for all $T>0$.

Proof: For fixed $m$, the Faedo-Galerkin approximation

$$
U_{m}=\sum_{i=1}^{m} g_{i m}(t) W_{i}
$$

of the solution $U$ of (2.2) is defined by the finite-dimensional system of nonlinear ordinary differential equations for $\operatorname{gim}(t)$ given by

$$
\begin{aligned}
\frac{d U_{m}}{d t}+L U_{m}+P_{m} B\left(U_{m}\right)+C U_{m} & =P_{m} F \\
U_{m}(0) & =P_{m} U_{0}
\end{aligned}
$$

where Pm is the orthogonal projection in $\Upsilon, D\left(L^{\frac{1}{2}}\right), D\left(L^{\frac{-1}{2}}\right)$ and $D(L)$ onto the space spanned by the $m$ eigenfunctions of $L$ given in (2.5). Let $X$ be the finite-dimensional Hilbert space spanned by the $m$ eigenfunctions of $\mathrm{L}$ given in (2.5) with inner product $((.,)$.$) induced by D(L)$. Consider a closed ball of radius given by $(2.23)$ contained in $D(L)$. Integration of (2.24) and the above a priori energy type estimates provide a continuous mapping defined by the integral of (2.24) from the closed ball of radius $\rho_{2}^{2}$ given by (2.23) into itself. Thus, by the Leray-Shauder principle [26, 36] the finite-dimensional system of nonlinear ordinary differential equations (2.24) has at least one solution Um inside the ball of radius $\rho_{2}^{2}$. The passage to the limit

$$
m \rightarrow \infty \text { and } T_{m} \rightarrow T=\infty
$$

follows from the following a priori estimates: Utilizing the differential inequality (2.15) provides

$$
\begin{gathered}
\frac{d}{d t}\left\|U_{m}\right\|^{2}+\alpha \lambda_{1}\left\|U_{m}\right\|^{2} \\
\leq \frac{1}{\alpha}\left(\frac{R o}{F r^{2}}+\frac{1}{R_{o}}\right)^{2}
\end{gathered}
$$

which implies Um remains bounded in

$$
L^{\infty}((0, T) ; \Upsilon) \cap L^{2}\left(0, T ; D\left(L^{\frac{1}{2}}\right)\right)
$$

This useful result combined with the weak compactness implies there is a subsequence also denoted by Um and

$$
U \in L^{\infty}(0, T ; \Upsilon) \cap L^{2}\left(0, T ; D\left(L^{\frac{1}{2}}\right)\right)
$$

such that 


$$
U_{m} \rightarrow \begin{cases}U \in L^{2}\left(0, T ; D\left(L^{\frac{1}{2}}\right)\right) & \text { weakly } \\ U \in L^{\infty}(0, T ; \Upsilon) & \text { weak-star. }\end{cases}
$$

Using (2.7) and (2.10), we obtain the boundeness of $B\left(U_{m}\right)$ and $P_{m} B\left(U_{m}\right)$ in $L^{2}\left(0, T ; D\left(L^{\frac{-1}{2}}\right)\right)$. Furthermore, from (2.24), $\frac{d U_{m}}{d t}$ is also bounded in $L^{2}\left(0, T ; D\left(L^{\frac{-1}{2}}\right)\right)$. Utility of this result and weak compactness yield

$$
\frac{d U_{m}}{d t} \rightarrow \frac{d U}{d t} \in L^{2}\left(0, T ; D\left(L^{\frac{-1}{2}}\right)\right)
$$

weakly. The assertion of Lebesgue dominated convergence theorem as well as the above convergence results yield $U_{m} \rightarrow U \in L^{2}(0, T ; \Upsilon)$ strongly. We pass the limit in (2.24) and obtain the required result (2.2). We obtain

$$
U \in C([0, T] ; \Upsilon) \cap L^{2}\left(0, T ; D\left(L^{\frac{1}{2}}\right)\right) .
$$

Similarly, from (2:18) and (2:21) we conclude that if $U_{0} \in D\left(L^{\frac{1}{2}}\right)$ then by the Faedo-Galerkin technique

$$
U \in C\left([0, T] ; D\left(L^{\frac{1}{2}}\right)\right) \cap L^{2}(0, T ; D(L))
$$

for all $T>0$. According to Gronwall's inequality, uniqueness and continuity with respect to initial conditions of solution $U(t)$ follows from considering the difference between two solutions of the initial-value problem (2.2) and employing a priori estimates (2.17-2.23). Uniqueness and continuity with respect to initial conditions of solution $U(t)$ generate a dynamical system which is prescribed by continuous solution operators $S(t), t \in \mathbb{R}$ defined by

$$
S(t) U_{0}=U(t) \equiv S\left(U_{0}, t\right)
$$

satisfying the group property

$$
\begin{gathered}
S(t) U(s)=S(s) U(t) \\
S(0) U=U \quad \forall t, s \in \mathbb{R} .
\end{gathered}
$$

That the solution operators $S(t)$ are continuous is a consequence of the continuity of $U(t)$ in time and in initial conditions. The group property is a consequence of the injectivity of the solution operators which is equivalent to the backward uniqueness of solution for the initial-value problem (2.2). Next, we turn our attention to establish uniqueness, continuity and Frechet differentiability with respect to initial conditions of solution $U(t)$ for the initial-value problem (2.2). As in the previous section, the technique of the proof uses the linearization of the evolution equation (2.2) about the difference of two given solutions. For the sake of completeness, we repeat the analysis here. Consider u and v satisfying the evolution equation (2.2) corresponding to the initial conditions $u(0)=u_{0}$ and $v(0)=v_{0}$, respectively. The precise analysis of the solution difference $w=v-w$ yields

$$
\begin{array}{rlrl}
\frac{d w}{d t}+L w+N(v)-N(u) & = & 0 \\
w(0) & =v_{0}-u_{0}
\end{array}
$$


Where

$$
\begin{aligned}
N(v)-N(u) & =B(v, v)-B(u, u)+C w \\
& =B(v, w)+B(w, u)+C w \\
& =B(u, w)+B(w, u)+B(w, w)+C w .
\end{aligned}
$$

The linearization of (2.27) along the dynamical system trajectory satisfies the variational equation

$$
\begin{aligned}
\frac{d \Psi}{d t}+L \Psi+l_{0}(t) \Psi & =0 \\
\Psi(0) & =v_{0}-u_{0}=\xi
\end{aligned}
$$

Where $l_{0}(t) w=B(u(t), w)+B(w, u(t))+C w$ is linear bounded operator from $D\left(L^{\frac{1}{2}}\right)$ to $D\left(L^{\frac{-1}{2}}\right)$. Consequently, the equation of variation is a linear nonautonomous ordinary differential system. We shall be concerned with the difference $\varphi=w-\Psi$ and the following is valid

$$
\begin{aligned}
\frac{d \varphi}{d t}+L \varphi+l_{0}(t) \varphi+l_{1}(t ; w(t)) & =0 \\
\varphi(0) & =0
\end{aligned}
$$

Where $l_{1}(t ; w(t))=B(w, w)$. The remarkable feature of the last equality is that from (2.28), we have $N(v)-N(u)=l_{0}(t) w+l_{1}(t ; w) \cdot$ We are now in a position to state the keystone result:

Proposition 2.1.2 If $u_{0} \in \Upsilon$ and $u$ is the unique solution of (2.2) then the variational equation (2.29) has a unique solution $\Psi$ satisfying

$$
\Psi \in C([0, T] ; \Upsilon) \cap L^{2}\left(0, T ; D\left(L^{\frac{1}{2}}\right)\right)
$$

for all $T>0$. Moreover, the dynamical system $u_{0} \rightarrow S(t) u_{0}$ is Frechet differentiable in $\Upsilon$ with differential $L\left(t, u_{0}\right): \xi \rightarrow \Psi(t)$ given by the solution of the variational equation.

Proof: Existence and uniqueness of solution to (2.29) is a consequence the Faedo-Galerkin technique and the use of a priori estimates together with the Gronwall's inequality. Next, we exhibit a series of a priori estimates which will be required in establishing the above proposition. Taking the inner product of (2.27) with $\mathrm{w}$ and application of Cauchy-Schwarz inequality, Young's inequality and Sobolev inequalities yield

$$
\frac{d}{d t}\|w\|^{2}+\left\|L^{\frac{1}{2}} w\right\|^{2} \leq k^{2}\|w\|^{2},
$$

where $k=c_{1} \rho_{2}$ and $\rho_{2}$ is given in the estimate (2.22). According to the Gronwall's inequality the following is valid

$$
\|v(t)-u(t)\|^{2} \leq \exp \left(k^{2} T\right)\left\|v_{0}-u_{0}\right\|^{2}
$$


$\forall t \in(0, T)$. The sharp a priori estimate (2.32) yield forward uniqueness and continuity with respect to initial conditions of solution. Furthermore, from (2.31) we obtain a priori energy type estimate

$$
\int_{0}^{t}\left\|L^{\frac{1}{2}} w(s)\right\|^{2} d s \leq \exp \left(k^{2} T\right)\left\|v_{0}-u_{0}\right\|^{2} .
$$

By the utility of the inner product of (2:30) with ' we obtain the differential inequality

$$
\frac{d}{d t}\|\varphi\|^{2}+\frac{1}{2}\left\|L^{\frac{1}{2}} \varphi\right\|^{2} \leq k^{2}\|\varphi\|^{2}+\frac{c_{1}^{2}}{\lambda_{1}}\left\|L^{\frac{1}{2}} w(t)\right\|^{3}
$$

We repeat the construction using Gronwall's inequality and find a priori estimate

$$
\|\varphi(t)\|^{2} \leq \int_{0}^{T}\left\|L^{\frac{1}{2}} w(s)\right\|^{3} d s
$$

$\forall t \in(0, \infty)$. Consequently we obtain the bound

$$
\|\varphi(t)\|^{2} \leq \frac{c_{1}^{2}}{\lambda_{1}} \exp \left(3 k^{2} T / 2\right)\left\|v_{0}-u_{0}\right\|
$$

which implies the needed result

$$
\frac{\left\|S(t) v_{0}-S(t) u_{0}-L\left(t, u_{0}\right) \cdot\left(v_{0}-u_{0}\right)\right\|^{2}}{\left\|v_{0}-u_{0}\right\|^{2}}=o\left(\left\|v_{0}-u_{0}\right\|\right)
$$

As $v_{0} \rightarrow u_{0}$. Hence, the dynamical system $S(t): u_{0} \rightarrow u(t)$ is Frechet differentiable at $u_{0} \in \Upsilon$.

Next, we examine the problem of proving backward uniqueness of solution for the initialvalue equation (2.2) that establish the injectivity properties and group properties of the solution operators $S(t)$. The backward uniqueness of solution is proved in approximately the same way as Frechet differentiability result using the standard log-convexity method as in the previous section. For the sake of completeness of presentation we cover the proof of the injectivity of solution operators here. In order to motivate this objective, consider two solutions $\mathrm{u}$ and $\mathrm{v}$ of the initial-value problem such that at time $t=t_{*}$ both $u$ and $v$ satisfy (2.2) for $t \in\left(t_{*}-\epsilon, t_{*}\right)$ and $u\left(t_{*}\right)=v\left(t_{*}\right)$. Given that this hypothesis is valid, backward uniqueness of the solution operators is accomplished if $u(t)=v(t)$ for all time $t<t_{*}$ whenever the solutions are well-defined. Alternatively, suppose the solution operators satisfy

$$
S(t)=u(t+s) \forall \quad t>0 \quad s \in \mathbb{R}
$$

then for $\tau \in(0, \epsilon)$, the problem of backward uniqueness is whether the following hold:

$$
S(\tau) u\left(t_{*}-\tau\right)=S(\tau) v\left(t_{*}-\tau\right) \Rightarrow u\left(t_{*}-\tau\right)=v\left(t_{*}-\tau\right)
$$

which is the injectivity of the solution operators $S(\tau)$. The following significant result on the injectivity of the solution operators is valid:

Proposition 2.1.3 Suppose the following hold

$$
u, v \in L^{\infty}\left(0, T ; V=D\left(L^{\frac{1}{2}}\right) \cap L^{2}(0, T ; H=D(L))\right.
$$


then the difference $w=v-u$ satisfies the differential equation (2.27) for $t \in(0, T)$ and the solution operators $S(t)$ are injective.

We give a sketch of an elegant proof due to Temam [49].

Proof: We will use the notation $\varphi=\varphi(t)$ for the quotient

$$
\begin{gathered}
\varphi=\frac{\|\varphi(t)\|_{V}^{2}}{\|\varphi(t)\|_{H}^{2}} \\
=\frac{(L \varphi(t), \varphi(t))}{(\varphi(t), \varphi(t))} .
\end{gathered}
$$

Invoking Cauchy-Schwarz inequality, Young's inequality, Sobolev inequalities, continuity properties of the nonlinear operator $B(w, w)+C w$ developed above and the fact that $(L w-\varphi w, w)=0$ we obtain

$$
\begin{gathered}
\frac{1}{2} \frac{d}{d t} \varphi=\frac{\left.\left(\frac{d w}{d t}, w\right)\right)}{\|w\|^{2}}-\frac{\|w\|_{V}^{2}}{\|w\|^{4}}\left(\frac{d w}{d t}, w\right) \\
=\frac{1}{\|w\|^{2}}\left(\frac{d w}{d t}, L w-\varphi w\right) \\
=\frac{1}{\|w\|_{V}^{2}}(N(v)-N(u)-L w, L w-\varphi w) \\
=-\frac{\|L w-\varphi w\|^{2}}{\|w\|^{2}}+\frac{1}{\|w\|^{2}}(L w, N(v)-N(u)) \\
\leq-\frac{\|L w-\varphi w\|^{2}}{2\|w\|^{2}}+\frac{\|N(v)-N(u)\|^{2}}{2\|w\|^{2}} \\
\leq-\frac{\|L w-\varphi w\|^{2}}{2\|w\|^{2}}+k^{2} \varphi
\end{gathered}
$$

where $N(v)-N(u)$ is given in (2.28) and the function $k(t)=k_{1}(t)+B(w, w)+C w$ is defined by the mapping

$$
k_{1}(t): t \rightarrow c\left(\|u(t)\|_{V}^{\frac{1}{2}}\|L u(t)\|^{\frac{1}{2}}+\|v(t)\|_{V}^{\frac{1}{2}}\|L v(t)\|^{\frac{1}{2}}\right) \in L^{2}(0, T) .
$$

Utility of Gronwall's inequality into (2.36) gives

$$
\varphi(t) \leq \varphi(0) \exp \left(2 \int_{0}^{t} k^{2}(s) d s\right) \quad t \in(0, T)
$$

Thus, if the difference $w=v-u$ satisfies the above conditions and

$$
w(\tau)=0 \quad \Rightarrow \quad w(t)=0, \quad t \in[0, T]
$$

We proceed by contradiction. Hence, suppose that $\left\|w\left(t_{0}\right) \neq 0\right\|$ for $t_{0} \in[0, T)$. As a result by continuity we have $\|w(t) \neq 0\|$ on some open interval $\left(t_{0}, t_{0}+\epsilon\right)$ and we employ the notation $t_{*} \leq T$ for the largest time for which

$$
w(t) \neq 0 \quad \forall t \in\left[t_{0}, t_{*}\right) .
$$


It follows that $w\left(t_{*}\right)=0$. In the interval $\left[t_{0}, t_{*}\right)$ the mapping given by $t \rightarrow \log \|w(t)\|$ is well-defined and the fact that $w(t)$ satisfies the differential equation we obtain

$$
\frac{d}{d t} \log \frac{1}{\|w\|} \leq 2 \varphi+k^{2}
$$

Consequently for the time $t \in\left[t_{0}, t_{*}\right)$ the following is valid:

$$
\log \frac{1}{\|w\|} \leq \log \frac{1}{\left\|w\left(t_{0}\right)\right\|}+\int_{t_{0}}^{T} 2 \varphi(s)+k^{2}(s) d s
$$

which illustrates the boundedness of $\frac{1}{w(t)}$ from above as the time $t \rightarrow t_{*}$ from below and this is a contradiction. 


\section{Chapter 3. Nonlinear Stability of Solutions}

\subsection{Aspects of stability and attraction for the rotating Boussinesq equations with Reynolds stress}

In this section we consider nonlinear stability and attractors of solutions of rotating Boussinesq equations by adapting a priori estimates employed in establishing well-posedness and differentiability of solutions. One of the advantages of the results presented in this chapter is that they may be considered as more refined generalization of results such as (2.16) for the dissipation of flow energy and the entropy production provided by

$$
E(t)=\frac{1}{2}\|U(t)\|^{2}=\frac{1}{2} \int_{\Omega}\left(\underline{u}^{2}+\rho^{2}\right) d x,
$$

which is specification of energy and the entropy production in the $\mathrm{L}^{2}$-norm. Hence, the results of this section on nonlinear stability differ in form rather than in essence from their counterparts utilized in establishing well-posedness and differentiability of solutions. The problem of showing that the rest state of system (2:2) is nonlinearly stable is investigated using ideas developed in Galdi et al [19, 20]. An important quantity in analyzing whether the solution of an evolution equation is bounded in some suitable norm is that of Lyapunov functional or a priori energy type estimates of the system as we have already noted in establishing well-posedness. We will illustrate in the sequel a technique for constructing Lyapunov functionals with interpretations such as energy, enstrophy and entropy which are decreasing along solutions. The equations governing the flow of an incompressible stratified fluid under the Coriolis force induce damping mechanisms in the nature of viscosity, diffusion, stratification and rotation effects that manifest themselves in the evolution equation with the existence of Lyapunov functionals which are equivalent to some norm induced by the inner product of solution. Here we state a technique for constructing generalized Lyapunov functionals that furnish necessary and sufficient conditions for nonlinear stability. Specifically, the tools used are spectral properties of the linear operator and a priori estimates for the nonlinear operator. Now we recall definitions which are required in the formulation of the nonlinear stability criteria to be customized for use in the solutions of the rotating Boussinesq equations. Consider the following prototype initial-value problem

$$
\frac{d U}{d t}=L U+N(U), \quad U(0)=U_{0} .
$$

where $L U$ is linear operator and $N(U)$ is nonlinear operator in the sense that its Frechet derivative at zero vanishes. A self-adjoint linear operator $L$ for the initial-value problem (3.1) is called essentially dissipative if

$$
\begin{gathered}
(L U, U) \leq 0 \quad \forall \quad U \in D(L) \\
(L U, U)=0 \quad \Rightarrow \quad U=0
\end{gathered}
$$

The complement of essentially dissipative is called essentially non-dissipative. By the spectral theorem [19, 32], essential dissipativity is equivalent to the spectrum of $\mathrm{L}$ being nonnegative and zero not an eigenvalue. For every essentially dissipative operator L, the bilinear form 


$$
(U, W)_{L}=-(L U, W) \quad \forall U, W \in D(L)
$$

defines a scalar product in $\mathrm{D}(\mathrm{L})$. We denote by $\mathrm{HL}$ the completion of $\mathrm{D}(\mathrm{L})$ in the norm $\|\cdot\|_{L}$ given by

$$
\|U\|_{L}^{2}=-(L U, U) .
$$

According to the condition (3.2) on essential dissipativity of linear operators, we are in a position to state necessary and sufficient criteria for nonlinear stability using the Lyapunov functional

$$
J(U)=-(L U, U)
$$

and generalized energy flow energy and entropy production

$$
E(t)=\frac{1}{2}\left\{\|U\|^{2}+\|U\|_{L}^{2}\right\}
$$

for the paradigmatic example of initial-value problem (3.1).

Proposition 3.1.1 Suppose L is essentially dissipative and the nonlinear operator satisfies

$$
\|N U\|^{2} \leq c\|U\|_{L}^{2}\left(\|L U\|^{2}+\|U\|_{L}^{2}\right)
$$

where $c>0$. Then the rest state of system (3.1) is asymptotically stable, that is,

$$
J(U) \leq \frac{1}{\eta}\left\{2 E(0)\left(1+\frac{1}{\gamma \mu_{0}}\right) \exp \left[\frac{4(1-\gamma)}{\mu_{0} \gamma}\right]\right\} /(t+1),
$$

Whenever

$$
\gamma=(1-c E(0))>0
$$

where $\eta>0$ and $\mu_{0}=\frac{1}{2} \cdot$ Next assume L is essentially non-dissipative and the nonlinear operator satisfies

$$
\|N U\|^{2} \leq c J(U)\left(\|L U\|^{2}+\|U\|^{2}\right)
$$

$c>0$. Then the rest state is unstable in the sense of Lyapunov.

This proposition is proved in approximately the same way as the analogous fact for the finite-dimensional linear problem with the assistance of a priori energy type estimates that dominates the nonlinearity by linear elliptic operators.

Proof: Taking the inner product of the equation (3.1) with $U$ yields

$$
\frac{1}{2} \frac{d}{d t}(U, U)=(L U, U)+(N U, U) .
$$

Similarly, taking the inner product of the equation (3.1) with $L U$ gives

$$
\frac{1}{2} \frac{d}{d t}(L U, U)=(L U, L U)+(L U, N U) .
$$

Putting the estimates together provide the following equation for the evolution the generalized energy functional (3.5)

$$
\frac{d E}{d t}=(L U, U)+(N U, U)-(L U, L U)-(L U, N U) .
$$

Applying Cauchy-Schwarz inequality and Young's inequality in (3.10) yields 


$$
\begin{aligned}
\frac{d E}{d t} & \leq(L U, U)+\frac{3}{2}\|L U\|^{2}+\|U\|\|N U\|+\frac{1}{2}\|N U\|^{2} \\
& \leq-\mu_{0}\left(\|L U\|^{2}+|U|_{L}^{2}\right)+\|U\|\|N U\|+\frac{1}{2}\|N U\|^{2} .
\end{aligned}
$$

Substitution of a priori estimate (3.6) in (3.11) and the courtesy of the generalized energy functional (3.5) provides the differential inequality

$$
\begin{aligned}
\frac{d E}{d t} & \leq-\mu_{0}\left(\|L U\|^{2}+\|U\|_{L}^{2}\right)\left[1-c^{\frac{1}{2}}\|U\|_{L}\|U\|\left(\|L U\|^{2}+\|U\|_{L}^{2}\right)^{\frac{-1}{2}}-\frac{c}{2}\|U\|_{L}^{2}\right] \\
& \leq-\mu_{0}\left(\|L U\|^{2}+\|U\|_{L}^{2}\right)\left[1-\frac{c}{2}\|U\|_{L}^{2}\right] \\
& \leq-\mu_{0}\left(\|L U\|^{2}+\|U\|_{L}^{2}\right)[1-c E] .
\end{aligned}
$$

According to the inequality (3.12) and assumption (3.8) we obtain a priori energy estimate

$$
\mu_{0}\left(\|L U\|^{2}+\|U\|_{L}^{2}\right)(1-c E(0)) \leq-\frac{d E}{d t}
$$

which implies

$$
\int_{0}^{\infty} \mu_{0}\left(\|L U\|^{2}+\|U\|_{L}^{2}\right)\left(1-\frac{c}{\mu_{1}} E(0)\right) d t \leq 2 E(0) .
$$

In this respect it should be noticed that the following a priori energy type estimate holds

$$
\int_{0}^{\infty}\left(\|L U\|^{2}+\|U\|_{L}^{2}\right) d t \leq \frac{2 E(0)}{\mu_{0} \gamma} .
$$

We proceed by recalling the useful property

$$
-\frac{1}{2} \frac{d}{d t}(L U, U)=-(L U, L U)+(L U, N U)
$$

and invoking the Cauchy-Schwarz inequality, Young's inequality and a priori estimate (3.6) which yield the following differential inequality

$$
\frac{d}{d t}\|U\|_{L}^{2} \leq 2 c\|U\|_{L}^{2}\left(\|L U\|^{2}+\|U\|_{L}^{2}\right)
$$

In order to establish the hypotheses of Gronwall's inequality we note that (3.13) may be restated equivalently as follows:

$$
\frac{d \Phi}{d t} \leq h_{1}(t)+\frac{2 c}{\mu_{1}} h_{2}(t) \Phi
$$

Where

$$
\begin{gathered}
\Phi(t)=\|U\|_{L}^{2}(t+1), \\
h_{1}(t)=\|U\|_{L}^{2},
\end{gathered}
$$


And

$$
h_{2}(t)=\|L U\|^{2}+\|U\|_{L}^{2} .
$$

Thus, the following holds

$$
\Phi(t) \leq \exp \left[2 c \int_{0}^{t} h_{2}(s) d s\right]\left\{\Phi(0)+\int_{0}^{t} \exp \left[-2 c \int_{0}^{s} h_{2}(\alpha) d \alpha\right] h_{1}(s) d s\right\}
$$

which is the assertion of Gronwall's inequality. Moreover, through the use of estimates from above we deduce the following a priori estimate that guarantee the hypotheses of the Gronwall's lemma are valid

$$
\int_{0}^{\infty} h_{1}(t) d t \leq \int_{0}^{\infty} h_{2}(t) d t \leq \frac{2 E(0)}{\mu_{0} \gamma} .
$$

Another application of relation (3.8) yields a priori estimate

$$
2 c \int_{0}^{\infty} h_{2}(t) d t \leq \frac{4 c E(0)}{\mu_{0} \gamma}=\frac{4(1-\gamma)}{\mu_{0} \gamma} .
$$

Consequently, we have

$$
\exp \left[\frac{2 c}{\mu_{1}} \int_{0}^{\infty} h_{2}(t) d t\right] \leq \exp \left[\frac{4(1-\gamma)}{\mu_{0} \gamma}\right]
$$

Similarly, putting the above estimates together we obtain the following finite energy solution

$$
\Phi(t) \leq 2 E(0)\left(1+\frac{1}{\gamma \mu_{0}}\right) \exp \left[\frac{4(1-\gamma)}{\mu_{0} \gamma}\right] .
$$

Substitution of $\Phi(t)=\|U\|_{1}^{2}(t+1)$ into (3.15) gives the desired nonlinear stability result (3.7) since $J(U) \leq \frac{1}{\eta}\|U\|_{1}^{2}$ whenever L is essentially dissipative. Hence, as a bonus we obtain the proof of Gronwall's lemma. The instability result holds by following the proof given in $[19,20]$.

For the rest states, asymptotic stability and attraction coincide. However, for more complicated invariant sets than rest states, an attractor may be considered as the stronger notion of stability than the one given above. The following proposition $[51,50,49,48,15,14,54,52,53,60]$ on the characterization of attractors and determining of the compactness of an $\omega$-limit set is required:

Proposition 3.1.2 Suppose that for $Q \subset H, Q \neq \emptyset$, and that for some $s>0$ the set $\cup_{t \geq s} S(t) Q$ is relatively compact in $H$. Then $\omega$-limit set of $Q$ denoted by $\omega(Q)$ is nonempty, compact and invariant.

Proof: The proposition is proven on page 20 in Temam [49] using properties of invariant sets.

The proposition is of central importance in the construction of attractors. In order to prove the major hypothesis that $\cup_{t \geq s} S(t) Q$ is relatively compact in $H$, it is adequate to illustrate that this set is bounded in a space V compactly imbed- 
ded in $H$ by employing the existence of the following injections

$$
D\left(L^{\frac{1}{2}}\right)=V \subset D(L) \subset \Upsilon=H \subset D\left(L^{\frac{-1}{2}}\right) .
$$

The density and compactness of the injections will find applications in the sequel when we prove existence of attractors for the initial-value problems.

The aim of this section is to establish nonlinear stability results for the rest state of rotating Boussinesq equations with Reynolds stress $(1.29$ - 1.31). In order to give nonlinear stability bounds that agree approximately with established paradigmatic example of initial-value problem, the functional formulation for the evolution equation of $\beta$-plane rotating Boussinesq equations with Reynolds stress is restated equivalently as follows:

$$
\begin{aligned}
\frac{d U}{d t}+L U+N(U) & =0 \\
U(0) & =U_{0}
\end{aligned}
$$

where $U=(u, v, w, \rho)$. The boundary conditions have been taken to be space-periodic as in the investigation of well-posedness of solution. Concerning the linear operator $L U$ and the nonlinear operator $N(U)$ we have the following appropriate setup:

$$
\begin{aligned}
& N(U)=B(U, U) \\
& L U=T U+C U+S U-F U \\
& F U=-\left(0,0, \frac{\rho}{R o}, \frac{R o}{F r^{2}} w\right) \\
& C U=-\left(\begin{array}{l}
\frac{E k}{R o} \triangle \underline{u} \\
\frac{1}{E d} \triangle \rho
\end{array}\right)=\left(\begin{array}{l}
A_{l} \underline{u} \\
A_{l} \rho
\end{array}\right) \\
& B(U, U)=B(U)=\left(\begin{array}{l}
\underline{u} \cdot \nabla \underline{u} \\
\underline{u} \cdot \nabla \rho
\end{array}\right)=\left(\begin{array}{l}
\mathbf{b}_{l}(\underline{u}, \underline{u}) \\
\mathbf{b}_{l}(\underline{u}, \rho)
\end{array}\right) \\
& T U=-\left(\begin{array}{l}
\frac{E k}{R o} \triangle^{6} \underline{u} \\
\frac{1}{E d} \triangle^{6} \rho
\end{array}\right)=\left(\begin{array}{l}
A_{l}^{6} \underline{u} \\
A_{l}^{6} \rho
\end{array}\right) \\
& S=-\left(\frac{1+y \beta R o}{R o}\right)\left(\begin{array}{cccc}
0 & -1 & 0 & 0 \\
1 & 0 & 0 & 0 \\
0 & 0 & 0 & 0 \\
0 & 0 & 0 & 0
\end{array}\right) \forall U \in D(L)=D(T)
\end{aligned}
$$


From the above definition of the linear operator $L U$, we have

$$
(L U, U)=(T U, U)+(C U, U)+\left(\frac{1}{R o}+\frac{R o}{F r^{2}}\right) \int_{\Omega} \rho w d x
$$

which is a modification of the bilinear form (2:3) that was employed in establishing wellposedness of solution for the problem of rotating Boussinesq equations with Reynolds stress. As in the previous section, with the attendant relation (3.17) we are able to demonstrate a necessary and sufficient nonlinear stability criterion and hence the elegant power of energy methods is fully realized. For the sake of completeness, we repeat the analysis in this section for rotating Boussinesq equations with Reynolds stress. The first effort is then to let $\mathrm{R}$ and

$$
I(\underline{u}, \rho)
$$

be defined as follows

$$
1 / 2 R=\sup \left\{\int_{\Omega} \rho w d x\right\} / J(U)=\sup I(\underline{u}, \rho)
$$

where the supremum is taken for $U \in D(L)$. We shall compile various inequalities about the equation (3.16). Substituting

$$
\left|\int_{\Omega} \rho w d x\right| \leq\left(\|\underline{u}\|^{2}+\|\rho\|^{2}\right) / 2
$$

into (3.18) and utilizing the Poincare-Friedrichs inequality yields $R \geq G a$. The attendant relation (3.17) may be restated as follows

$$
(L U, U)=\left(1+\left(\frac{1}{R o}+\frac{R o}{F r^{2}}\right) I(\underline{u}, \rho)\right) J(U),
$$

with the generalized energy functional $J(U)$ defined in (3.4) given by the Lyapunov functional

$$
J(U)=(T U, U)+\frac{E k}{R o}\|\nabla \underline{u}\|^{2}+\frac{1}{E d}\|\nabla \rho\|^{2}
$$

It is appropriate at this point to note that if $\left(\frac{1}{R o}+\frac{R o}{F r^{2}}\right)<2 R$ then the following is valid

$$
-(L U, U) \leq\left(-1+\left(\frac{1}{2 R R o}+\frac{R o}{2 R F r^{2}}\right)\right) J(U)<0
$$

and in this case $-L$ is essentially dissipative. Additionally, the following inequalities hold

$$
\left(1-\left(\frac{1}{2 R R o}+\frac{R o}{2 R F r^{2}}\right)\right) J(U) \leq\|U\|_{L}^{2} \leq\left(1+\left(\frac{1}{\pi^{2} R o}+\frac{R o}{\pi^{2} F r^{2}}\right)\right) J(U)
$$

which shows that $\|U\|_{L}^{2}$ is topologically equivalent to $J(U)$. 
The next step is to note that if $\left(\frac{1}{R o}+\frac{R o}{F r^{2}}\right)>2 R$ then there is a sequence $\left\{U_{n}\right\} \subset \Upsilon$ such that

$$
\lim _{n \rightarrow \infty} I\left(\underline{u}_{n}, \rho_{n}\right)=1 / 2 R \text {. }
$$

Hence given $\epsilon<\left(\frac{1}{2 R}-\left(\frac{1}{R o}+\frac{R o}{F r^{2}}\right)\right)$, there exists $\mathrm{U}$ such that

$$
-(L U, U) \geq\left[-1+\left(\frac{1}{R o}+\frac{R o}{F r^{2}}\right)\left(\frac{1}{2 R}-\epsilon\right] J(U)>0,\right.
$$

and in this case $-L$ is essentially non-dissipative. We require the following lemma on the spectrum of $-L$ whose validity may be proved by using the technique of proof similar to those given above:

Lemma 3.1.3 $\sigma(-L) \subseteq(-\infty, 0] \cup \amalg$, where II is either empty or an at most denumerable set consisting of isolated, positive eigenvalues $\lambda_{n}=\lambda_{n}\left(\frac{1}{R o}+\frac{R o}{F r^{2}}\right)$ with finite multiplicity such that

$$
\infty>\lambda_{1} \geq \lambda_{2} \geq \ldots \geq \lambda_{n} \geq \ldots
$$

clustering only at zero.

Next, we proceed to illustrate that suitable a priori estimates for the nonlinear operator $N(U)$ are available. Putting estimates (2.6) and (2.8) together into the inequality (2.10) yield the desired bounds

$$
\begin{aligned}
\|N U\|^{2} & \leq(C U, U) c_{0}^{2}\left(\|C U\|^{2}+\|U\|^{2}\right) \\
& \leq c_{0}^{2}\left\|C^{\frac{1}{2}} U\right\|\left(\lambda_{1}\left\|L^{\frac{1}{2}} U\right\|^{2}+\|U\|^{2}\right) \\
& \leq c_{0}^{2} \lambda^{\frac{-1}{2}}\|C U\|\left(\lambda_{1}\left\|L^{\frac{1}{2}} U\right\|^{2}+\|U\|^{2}\right) \\
& \leq c_{0}^{2} \lambda^{\frac{-1}{2}} \lambda_{1}^{\frac{1}{2}}\left\|L^{\frac{1}{2}} U\right\|\left(\lambda_{1}^{\frac{1}{2}}\|L U\|^{2}+\|U\|^{2}\right) \\
& \leq c_{0}^{2} c \lambda^{\frac{-1}{2}} \lambda_{1}^{\frac{1}{2}}\left\|L^{\frac{1}{2}} U\right\|\left(\|L U\|^{2}+\|U\|^{2}\right) \\
& =c_{0}^{2} c \lambda^{\frac{-1}{2}} \lambda_{1}^{\frac{1}{2}} J(U)\left(\|L U\|^{2}+\|U\|^{2}\right)
\end{aligned}
$$

for the nonlinearity $N(U)=B(U)$ where $c=\max \left\{\lambda_{1}^{\frac{1}{2}}, 1\right\}$. Thus, the purpose of proving Lyapunov instability aspect has been achieved. Moreover, when the following $\left(\frac{1}{R o}+\frac{R o}{F r^{2}}\right)<2 R$ holds, we observe the topological equivalence of the Lyapunov functional $J(U)$ with the norm $\|U\|_{L}^{2}$ given by the inequality (3.22). In this case, putting the estimates 
(2.6) and (3.25) together and invoking the equivalence relation (3.22) gives the needed a priori estimate for the nonlinearity

$$
\begin{aligned}
\|N U\|^{2} & \leq c_{0}^{2} c \lambda^{\frac{-1}{2}} \lambda_{1}^{\frac{1}{2}} J(U)\left(\|L U\|^{2}+\|U\|^{2}\right) \\
& \leq c_{1} c_{0}^{2} c \lambda^{\frac{-1}{2}} \lambda_{1}^{\frac{1}{2}}\|U\|_{L}^{2}\left(\|L U\|^{2}+\lambda_{1}^{-1}\|U\|_{L}^{2}\right)
\end{aligned}
$$

where $c_{1}=\left(1-\left(\frac{1}{2 R R o}+\frac{R o}{2 R F r^{2}}\right)\right)^{-1}$. According to the sharp a priori estimate (3.25), aspect of nonlinear stability provided by the inequality (3.6) is satisfied. Hence, energetic stability criteria for necessary and sufficient for nonlinear stability of the rest state of $\beta$-plane rotating Boussinesq equations with Reynolds stress (3.16) or equivalently (1.29 1.31) hold with the energy and entropy production provided by the following:

$$
E(t)=\frac{1}{2}\left\{\|U\|^{2}+J(U)+2 R \int_{\Omega} \rho w d x\right\}
$$

which is specification of energy and the entropy production in the Sobolev H1-norm if the criterion $G a \leq R<\left(\frac{1}{R o}+\frac{R o}{F r^{2}}\right)$ is valid. The result serves as the archetype for constructing generalized Lyapunov functionals with interpretations such as energy and entropy which are decreasing along solution of the system. Furthermore, we observe that in the craft of nonlinear stability, the equations governing the flow of an incompressible stratified fluid under the Coriolis force induce damping mechanisms in the nature of viscosity, diffusion, combined stratification and rotation effects that manifest themselves in the evolution equation with the existence of Lyapunov functionals which are equivalent to some fictitious energy or enstrophy or entropy. In the final analysis, it is reasonable in an attempt to give geophysical meaning to the above established nonlinear stability criteria to recall the roles of the nondimensional parameters employed in the construction of the above generalized Lyapunov functional: Ro is the Rossby number which compares the inertial term to the Coriolis force; $F r$ is the Froude number which measures the importance of stratification; Ek is the Ekman number which measures the relative importance of frictional dissipation; Ed is the nondimensional eddy diffusion coefficient. And the geophysically relevant ratio $\frac{F r^{2}}{R o}$ measures the significant inuence of combined rotation and stratification to the dynamics of the flow. It is crucial to note that the techniques employed in developing the nonlinear stability criteria have been completed without carrying out closed form wave solutions for mesoscale and synoptic eddies. The presence of stratification and Reynolds stress introduces some new considerations that need special attention since in this more general setting, exact close form solutions need not be easily accessible. Thus, most cases of oceanographic or meteorological interest such as vortex spin-down, the decay of energy in the nonlinear stability criteria need not account for the dissipation of energy and enstrophy for mesoscale and synoptic wave motions. However, we consider the energetic stability criteria as a first effort towards elucidating such geo-physical phenomena. Furthermore, we observe that the nonlinear stability criteria may give the possibility of a sharper examination of the numerical approximation of the solu- 
tion in the sense of the Lax equivalence theorem: a designed finite-difference scheme to an initial-value problem converges to the solution with the rate of convergence specified by the order of accuracy of the scheme.

Next, we focus our attention to the development of results for the attractor of the $\beta$-plane rotating Boussinesq equations with Reynolds stress (1.29-1.31) for both the three-dimensional and two-dimensional space variables. We show existence of the attractor for the dynamical system and proceed by customizing the splendid techniques employed in [11, 12, $49,48,15,14]$ which have been summarized in the above proposition. Concerning the results for the attractor of the general three-dimensional space variables initial-value problem for viscous $\beta$-plane rotating Boussinesq equations with Reynolds stress we infer from the more refined a priori estimates (2:23) that the attractor for (2:2) is provided by the $\omega$-limit set of $Q=B_{2 \rho_{2}}$,

$$
A=\omega(Q)=\cap_{s \geq 0} C l\left(\cup_{t \geq s} S(t) Q\right)
$$

where $B_{2 \rho_{2}}$ denotes an open ball in phase space of radius $2 \rho_{2}$, which depends on the geophysically relevant parameters. The closure is taken in the Hilbert space $\Upsilon$. Utility of the group property and continuity of the solution operators $S(t)$ defined for all time $t \in \mathbb{R}$, gives the following invariance property of the above established attractor:

$$
S(t) A=A, \quad \forall t \in \mathbb{R} .
$$

Consequently, examining the expression for the invariance of the attractor we observe that the attractor for the three-dimensional space variables initial-value problem for viscous $\beta$-plane ageostrophic equations with Reynolds stress is both positively and negatively invariant and consists of orbits or trajectories that are defined for all $t \in \mathbb{R}$, In the derivation of $\beta$-plane rotating Boussinesq equations with Reynolds stress, it was essential to note that the additive decomposition of the rotating Boussinesq equations quantities into coherent and incoherent terms and consideration of the averaging operator to obtain the Reynolds stress fields or wind stress fields resulted in a set of evolution equations that were not closed. In order to close the set of equations, we adopted the Pedlosky closure protocols.

\section{ACKNOWLEDGMENTS}

This research project is dedicated to Prof.Dr. Natalia Copelovici Sternberg, who taught me mathematical modelling with differential equations (ordinary differential equations, partial differential equations, stochastic differential equations, and functional differential equations) at Clark University, Worcester, Massachusetts, USA. I am deeply grateful for her expressions of encouragement and support and her generosity of spirit and fundamental good nature have inspired me. This masterly exposition and encyclopedic presentation of the nonlinear dynamics of rotating stratified fluid flows was done in collaboration with my students at University of Limpopo and I thank them for their assistance on matters de modus vivendi. Most of all I must thank my lovely wife and persuaded companion, Raesibe, who has read the manuscript and given invaluable information, inspiration, insight, and intuition. Her love and patience enabled her to be understanding in the face of my increased absence from her and our children, Thapelo and Seageng, while completing this report. 


\section{References}

[1] D.J. ACHESON, Elementary fluid dynamics, Clarendon Press, Oxford, 1990.

[2] R.A. ADAMS, Sobolev spaces, Academic Press, New York, 1975.

[3] S. AGMON AND L. NIRENBERG, Lower bounds and uniqueness theorems for solutions of differential equations in a Hilbert space, Comm. Pure Appl. Math, 20 (1967), 207-229.

[4] H. B AER AND K. STEPHAN. Heat and mass transfer, transl. by Janepark N., Springer-Verlag, Inc., New York, 1998.

[5] S.H. B ALASURIYA, Barriers and transport in unsteady flows: a Melnikov approach, SIAM, Philadelphia, 2017.

[6] G.K. BATCHELOR. Introduction to fluid dynamics. Cambridge University Press, Cambridge, 1967.

[7] A.J. BOURGEOIS AND J.T. BEALE, Validity of the quasigeostrophic model for large-scale flow in the atmosphere and ocean, SIAM J. Math. Anal. 25 (4) 1994:1023-1068.

[8] C. CANUTO, M.Y. HUSSAINI, A. QUARTERONI, AND T.A. ZANG, Spectral methods in fluid dynamics, Springer-Verlag, Inc., New York, 1988.

[9] A. CONSTANTIN, Nonlinear water waves with applications to wave-current interactions and tsunamis, CBMS regional conference, SIAM, Philadelphia, 2011.

[10] B. CUSHMAN-ROISIN, Introduction to geophysical fluid dynamics, Prentice-Hall, Inc., Englewood Cliffs, New Jersey, 1994.

[11] C.M. DAFERMOS, Contraction semigroups and trend to equilibrium in continuum mechanics, Springer-Verlag, Inc., New York, 1976.

[12] C.M. DAFERMOS, Hyperbolic conservation laws in continuum physics, 3rd ed., Springer-Verlag, Inc., New York, 2010.

[13] F. DUMORTIER, H. KOKUBU, AND H. OKA, A degenerate singularity generating geometric Lorenz attractors, Ergod. Th. Dynam. Sys. 15 (1995) 833-856.

[14] C. FOIAS, O. MANLEY AND R. TEMAM, Attractors for the Bernard problem: Existence and physical bounds on their fractal dimension, Nonlinear Anal. 11 (1987), 939-967.

[15] C. FOIAS, G. SELL AND R. TEMAM, Inertial manifolds for nonlinear evolutionary equations, J. Differential Equations 73 (1988), 309-353.

[16] S.J. FRIEDLANDER, Lectures on stability and instability of ideal fluid, Institute of Advanced Studies, Princeton University, Princeton, 1999.

[17] S.J. FRIEDLANDER, Introduction to the mathematical theory of geophysical fluid dynamics, North Holland, New York, 1980.

[18] G.P. GALDI, An introduction to the mathematical theory of the Navier-Stokes equations,

Springer-Verlag, Inc., New York, 1994.

[19] G.P. GALDI AND M. PADULA, A new approach to energy theory in the stability of fluid motion, Arch. Rational Mech. Anal. 110 (1990), 187-286.

[20] G.P. GALDI AND S. RIONERO, Weighted energy methods in fluid dynamics and elasticity, Springer-Verlag, Inc., New York, 1985.

[21] J. GUCKENHEIMER AND P. HOLMES, Nonlinear oscillations, dynamical systems and bifurcation of vector fields, Springer-Verlag, Inc., New York, 1983.

[22] M.E. GURTIN, An introduction to continuum mechanics, Academic Press, Inc., San Diego, 1981.

[23] J.K. HALE AND H. KOCAK, Dynamics and bifurcations, Springer-Verlag, Inc., New York, 1991.

[24] J.K. HALE AND S.M. VERDUYN LUNEL, Introduction to functional differential equations, Springer-Verlag, Inc., New York, 1993.

[25] J.K. HALE AND S.-N. CHOW, Methods of bifurcation theory, Springer-Verlag, Inc.,New York, 1982.

[26] J.K. HALE, Ordinary differential equations, Wiley-Interscience, New York, 1969.

[27] G. HALLER, Chaos near resonance, Springer-Verlag, Inc., New York, 1999.

[28] G. HALLER AND A.C. POJE, Finite time transport in aperiodic ows, Physica D 83 (1998) 353-380.

[29] P. HARTMAN, Ordinary differential equations, SIAM, Philadelphia, 2002.

[30] F. JOHN, Partial differential equations, 4th ed., Springer-Verlag, Inc., New York, 1971.

[31] C.K.R.T. JONES, Session on dynamical systems: geometric singular perturbation theory, C.I.M.E. Lectures, 1994.

[32] T. KATO, Perturbation theory for linear operators, Springer-Verlag, Inc. New York, 1966.

[33] R.E. KHAYAT, Chaos and o verstability in the thermal convection of viscoelastic fluids, J. Non-Newtonian Fluid Mech. 53 (1994) 227-255.

[34] A. MAJDA, Introduction to PDE and waves for the atmosphere and ocean, Courant Institute of Mathematical Sciences, New York University, New York, 2003. 
[35] A. MATSUMURU AND T. NISHIDA, The initial-boundary value problem for the equations of motion of general fluids, North-Holland Publishing Co., New York 10 (1982).

[36] O.A. LADYZHENSKAYA, The mathematical theory of viscous incompressible flow, transl. by R.A. Silverman and J. Chu, Gordon and Breach Science Publishers, New York, 1969.

[37] J.L. LUMLEY, G. BERKOOZ, AND P. HOLMES, Turbulence, coherent structures, dynamical systems and symmetry, Cambridge Uni versity Press, Cambridge, 1996.

[38] J.L. LUMLEY, ed., Turbulence at the crossroads, Springer-Verlag, Inc., New York, 1990.

[39] J. PEDLOSKY. Geophysical fluid dynamics, 2nd ed., Springer-Verlag, Inc., New York, 1987.

[40] H.-O. PEITGEN, H. JURGENS, AND D. SAUPE, Chaos and fractals: new frontiers of science, Springer-Verlag, Inc., New York, 1992.

[41] B.D. REDDY AND G.P. GALDI, Well-posedness of the problem of fiber suspension ows, J. Non-Newtonian Fluid Mech., 83 (1999) 205-230.

[42] B.D. REDDY, Introductory functional analysis, Springer-Verlag, Inc., New York, 1998.

[43] M. REED AND B. SIMON, Functional analysis, Academic Press, San Diego, 1980.

[44] H.L. ROYDEN, Real Analysis, Macmillan Publishing Co., New York, 1988.

[45] S. SMALE, Dynamics retrospective, Physica D 51 (1991) 267-273.

[46] E.A. SPIEGEL AND G. VERONIS, On the Boussinesq approximation for a compressible fluid, Astrophy. J. 131 (1960) $442-447$.

[47] S.H. STROGATZ, Nonlinear dynamics and chaos, Addison-Wesley, New York, 1994.

[48] R. TEMAM, Navier-Stokes equations and nonlinear functional analysis, CBMS regional conference, SIAM, Philadelphia, 1983.

[49] R. TEMAM, Infinite-dimensional dynamical systems in mechanics and physics, Springer-Verlag, Inc., New York, 1988.

[50] R. TEMAM, Navier-Stokes equations: Theory and Numerical Analysis, AMS Chelsea Ed., Providence, 2001.

[51] R. TEMAM, B. NICOLAENKO, C. FOIAS AND P. CONSTANTIN, Integral manifolds and inertial manifolds for dissipative partial differential equations, Springer- Verlag, Inc., New York, 1988.

[52] E.S. TITI AND C. CAO, Global well-posedness of the three-dimensional stratified primitive equations with partial vertical mixing turbulence diffusion, Communications in Mathematical Physics, 310 (2012) 537-568.

[53] E.S. TITI AND C. CAO, Global well-posedness of the three-dimensional viscous primitive equations of large scale ocean and atmosphere dynamics, Annals of Mathematics, 166 (2007) 245-267.

[54] E.S. TITI, On approximate inertial manifolds to the Navier-Stokes equations, J. Math. Anal. And Appl., 149 (1990) 540-557.

[55] M.S. TLADI, A Lagrangian and Eulerian analysis of a geophysical uid ow, submitted to Quaetiones Mathematicae.

[56] M.S. TLADI, On the qualitative theory of the rotating Boussinesq and quasigeostrophic equations, Quaetiones Mathematicae 40 (6) 2017: 705-737.

[57] M.S. TLADI, A geometric approach to differential equations, Lecture Notes, Department of Math. And Applied Math., University of Limpopo, 2009.

[58] M.S. TLADI, Well-posedness and long-time dynamics of beta-plane ageostrophic flows, Ph.D. Thesis, Department of Math. And Applied Math., Uni versity of Cape Town, 2004.

[59] A. TSINOBER AND H.K. MOFFATT, eds., Topological fluid mechanics, Cambridge University Press, Cambridge, 1990. [60] S. WANG, Attractors for the 3D baroclinic quasigeostrophic equations of large scale atmosphere, J. Math. Anal. And Appl. 165 (1992) 266-283.

[61] S. WANG, J.L. LIONS AND R. TEMAM, New formulations of the primitive equations of the atmosphere and applications, Nonlinearity 5 (1992), 237-288.

[62] S. WANG, J.L. LIONS AND R. TEMAM, On the equations of the large-scale ocean, Nonlinearity 5 (1992), 1007-1053.

[63] S. WIGGINS, Chaotic transport in dynamical systems, Springer-Verlag, Inc., New York, 1992.

[64] S. WIGGINS, Introduction to applied nonlinear dynamical systems and chaos, Springer-Verlag, Inc., New York, 1990.

[65] P.A. WORFOLK AND W. CRAIG, An integrable normal form for water waves in infinite depth, Physica D 84 (1995) 513-531.

[66] P.A. WORFOLK, J. GUCKENHEIMER, M. MYERS, F. WICKLIN AND A. BAK, Ds Tool: Computer assisted exploration of dynamical systems, Notices Amer. Math. Soc. 39 (1992), 303-309. 